\title{
Costs and benefits of dynamic trading in a lemons market
}

\author{
William Fuchs ${ }^{\mathrm{a}, \mathrm{b}, *}$, Andrzej Skrzypacz ${ }^{\mathrm{c}}$ \\ ${ }^{\text {a }}$ McCombs School of Business, UT Austin, United States of America \\ b Universidad Carlos III Madrid, Spain \\ c Graduate School of Business, Stanford University, United States of America
}

\section{A R T I C L E I N F O}

\section{Article history:}

Received 25 May 2018

Received in revised form 10 February 2019

Available online 13 March 2019

\section{JEL classification:}

D82

G14

G18

D02

D86

E58

E60

G01

\section{Keywords:}

Market design

Adverse selection

Trading frequency

Bankruptcy

\begin{abstract}
A B S T R A C T
We study a dynamic market with asymmetric information that creates the lemons problem. We compare efficiency of the market under different assumptions about the timing of trade. We identify positive and negative aspects of dynamic trading, describe the optimal market design under regularity conditions and show that continuous-time trading can always be improved upon if sellers are present at $t=0$. Instead, continuous trading is optimal if sellers arrive stochastically over time.
\end{abstract}

(c) 2019 The Authors. Published by Elsevier Inc. This is an open access article under the CC BY-NC-ND license (http://creativecommons.org/licenses/by-nc-nd/4.0/).

\section{Introduction}

When designing or regulating a market, an important variable to study is the frequency with which traders are allowed to trade or make offers to each other. In this paper we take the set of times the market is open, $\Omega$, as our only design or policy instrument and study how different timing protocols affect the equilibrium and welfare in a market with adverse selection.

In Akerlof (1970) the seller makes only one decision at the start of time: to sell the asset or not, $\Omega=\{0, \infty\}$. However, in practice, if the seller does not sell immediately, there are often future opportunities to trade. Delayed trade can be used by the market as a screen to separate low-value assets (those that sellers are more eager to sell) from high-value assets. As we show in this paper, dynamic trading creates costs and benefits for overall market efficiency. On the positive side, the screening via costly delay increases in some instances overall liquidity of the market: more types eventually trade in a dynamic trading market than in the static/restricted trading market. On the negative side, future opportunities to

\footnotetext{
We thank Ilan Kremer, Mikhail Panov, Christine Parlour, Aniko Öry, Brett Green, Marina Halac, Johanna He, Alessandro Pavan, Jean Tirole, Felipe Varas, Robert Wilson, and participants of seminars and conferences for comments and suggestions. Fuchs gratefully acknowledges support from the ERC Grant \#681575.

* Corresponding author.

E-mail addresses: wfuchs@gmail.com (W. Fuchs), skrz@stanford.edu (A. Skrzypacz).
} 
trade reduce the amount of early trade, making the adverse selection problem worse. There are two related reasons. First, fixing the time 0 price, $p_{0}$, buyers update positively about the value of the asset if the seller reject this price, leading to a higher future price. This makes it desirable for some seller types to wait. Second, the types who decide to wait are a better-than-average selection of the types that were supposed to trade at time 0 in a static model. Hence the average quality at time 0 falls, so $p_{0}$ must decrease. In turn, even more types decide to wait, reducing efficiency further.

Consider next the case where the seller is free to trade at any point in the interval $\Omega_{C}=[0, T]$ and all traders are present at $t=0$. When a privately informed seller can trade now or the next instant, it is really hard to screen types since the cost of waiting and trading the next instant is negligible. In this case, trade is smooth and a differential equation captures the speed at which types trade. If $T \rightarrow \infty$, asymptotically all types eventually trade but they do so at a slow pace. Next consider introducing a small closure of time $\Delta$ after the initial round of trade $\Omega^{E C} \equiv\{0\} \cup[\Delta, T]$. Now the sellers that were trading in $(0, \Delta)$ must decide if they trade earlier or later. Importantly, as some sellers start trading earlier, a virtuous circle takes place: as better types trade earlier, competition on the buyers' side implies that the price at $t=0$ must increase, but this in turn attracts even more sellers to trade early. This allows us to establish that if all the traders are present at $t=0$ then continuous trading is never optimal. A small departure from continuous trading leads to a Pareto improvement.

It is natural then to ask what happens as we increase $\Delta$. Consider the extreme case when $\Delta=T$ i.e., we allow just one opportunity to trade at time 0 and never again until $T$ (just as in Akerlof (1970) if $T=\infty$ ). In this case, there will be a large mass of sellers that trade at time 0 , but some seller types might prefer to hold on to their asset rather than receiving a low pooling price. For large $T$, this would not be a Pareto improvement over continuous trading, since some types that used to trade now don't do so anymore. Still, we show in Theorem 1 that, under a regularity condition (similar to what is used frequently in mechanism design), this is the optimal timing design in terms of maximizing expected gains from trade.

For both of these results and the recommended policy implications, it is important to identify what time 0 in the model corresponds to in practice. Our model shares this issue with any model in which time on the market plays a signaling role. In practice, identifying when gains from trade arise (say, because the seller is hit by a liquidity need) may not always be easy. Certain occasions may, nonetheless, provide a good proxy. For example, while working to stop the Deepwater Horizon oil spill, BP announced a plan to sell $\$ 30$ billion worth of assets in order to have the necessary liquidity to face the liabilities stemming from the accident. During the recent financial crisis, several financial institutions sold large portfolios of assets and minority stakes in other companies to strengthen their financial position and meet capital requirement regulations. ${ }^{1}$ Although not perfectly, the explosion of the oil well and the collapse of Lehman Brothers serve as natural candidates for time 0. Another good example is that of firms that enter into bankruptcy. As part of their reorganization, they commonly divest non-core assets and could use costly delay to signal the value of those assets. For such situations, our model suggests that to maximize expected gains from trade, there should be an organized auction early in the bankruptcy process with an obligation not to resell the asset for a period of time if the auction fails.

Our results also suggest that normal times, when there is no initial event and gains from trade arise stochastically over time, call for a laissez-faire approach. In Theorem 2 we show that in a stationary environment with sellers arriving at a Poisson rate over time (and in a linear-uniform case), discounted gains from trade are maximized by having the market continuously open. The cost of having some traders wait until the market opens surpasses the benefits highlighted above of restricting trading opportunities conditional on the seller being present. It is worth noting though that the cost of introducing small discrete trading intervals of size $\Delta$ is second order. Thus, if there are other first-order considerations such as high-frequency traders picking stale orders, it might still be beneficial to introduce some small trading restrictions.

Lastly, our findings that restrictions to future trading can improve welfare bring up an important practical issue: can the involved parties credibly commit to keeping the market closed in the future? As we point out in Remark 1 , one way to achieve such commitment is to make trades completely anonymous, so that past buyers could re-sell the asset if the market becomes active without their counterparties knowing whether they are facing the original seller or a previous buyer. If this were implemented, buyers would be discouraged from purchasing the asset after time zero since they would face additional adverse selection. As a result, the seller would not be able to get a higher price if he delays the transaction (unless he waits till the information arrives) and the gains from trade we describe in Theorem 1 would be realized.

\subsection{Related literature}

Our paper contributes to the literature on dynamic markets with adverse selection that includes Nöldeke and van Damme (1990), Swinkels (1999), Janssen and Roy (2002), Kremer and Skrzypacz (2007), Fuchs et al. (2016), and Daley and Green (2012). These papers characterize equilibria of trading games under different assumptions about information available in the market. While we share with these papers an interest in dynamic markets with asymmetric information, none of these papers focuses on timing as a design element. An exception, albeit using a search framework, is the work by Camargo and Lester (2014) who also study equilibrium dynamics with adverse selection and present an example where sunset provisions might be useful when introducing subsidies.

\footnotetext{
1 Merrill et al. (2012) show that the willingness to sell residential mortgage-backed securities by insurance companies can be partly explained by the severity with which their capital constraints were binding.
} 
In terms of results, our paper is most closely related to our earlier paper, Fuchs and Skrzypacz (2015) [FS]. There, we look at government interventions after events such as the recent financial crisis. ${ }^{2}$ There are two important differences: in FS we give the planner a richer set of instruments, and we assume $T=\infty$. We allow the planner to tax and subsidize trades differentially over time and endow it with an initial budget. Moreover, when we assume traders arrive over time, we allow the planner to regulate the prices in the market. When traders are present at time 0 , the extra power of the government is not too relevant. Indeed, as we show there (see FS Theorem 1), the government effectively uses taxes to close the market after an initial round of trade, similarly to Theorem 1 in this paper. There are many instances, such as in the design of trading platforms, in which a rich set of taxes and subsidies may not be an available instrument, and yet determining with which frequency players get to trade might be. In contrast to our previous work, this paper aims to highlight 'timing design' as a valuable form of market design.

From the perspective of proof techniques (use of mechanism design), both our papers are related to Samuelson (1984). He characterizes a welfare-maximizing mechanism in a static model subject to no-subsidy constraints. When $T=\infty$, this static mechanism design is mathematically equivalent to a dynamic mechanism design, since choosing probabilities of trade is analogous to choosing delay. Our analysis in this paper is more general since we also allow for both finite $T$ and for the case in which the arrival time is stochastic. In these cases, the models are no longer equivalent. Although our proof of Theorem 1 uses similar methods to Samuelson (1984), the problem changes in two important ways because we allow $T<\infty$. First, when calculating the gains from trade, now the alternative is to wait until time $T$ (or the stochastic arrival of information) and trade efficiently. This reduces the gains from trading at $t=0$. Second, there is an additional effect because the possibility of trading when information is revealed also changes the outside option of the seller. In turn, this changes the information rents that are captured by the different types. From an applied perspective, solving the model with temporary information asymmetry allows us to apply the model to situations such as stress tests or other forms or regulatory approval that might reveal the seller's private information.

When the seller is not present at time 0 , there is an important difference in results vis a vis FS. By regulating the price in the market to be equal to the pooling price of the period when there is only one trading possibility, the planner in FS is able to effectively eliminate any incentives to delay trade while allowing players to trade as soon as they enter the market. In this paper, the stationary instrument is a trading frequency $\Delta$, allowing trades at $\Delta, 2 \Delta, \ldots$ Increasing $\Delta$, increases the efficiency conditional on the seller being present, but at the cost that now the seller will arrive at $t \in(n \Delta,(n+1) \Delta)$. That would imply an efficiency loss of waiting for the first opportunity to trade. We show that this cost is larger than the benefits and thus it is optimal in this case to have the market continuously open.

Conceptually, we believe this paper is more directly related to papers where the timing of trades is a design element. The market microstructure literature (see Biais et al. (2005)) has also considered the question of how different trading protocols perform in the presence of adverse selection. That literature has mainly focused on stock markets where there are potentially many competing sellers, divisible assets and dispersed information. In this respect our work is related to Vayanos (1999) and Du and Zhu (2017) who focus on the effect of frequency of trade on the price impact of trades in imperfectly competitive markets. The differences between known and unknown timing of arrival have been also considered by Janssen and Karamychev (2002), who show that equilibria in dynamic markets with dynamic entry can be qualitatively different from markets with one-time entry if the "time on the market" is not observed by the market (see also Hendel et al. (2005) and Kim (2017) about the role of observability of past transaction/time on the market).

Finally, there is also recent literature on adverse selection with correlated values in models with search frictions (among others, Guerrieri et al. (2010), Guerrieri and Shimer (2014) and Chang (2018)). Rather than having just one market in which different quality sellers sell at different times, the separation of types in these models is achieved because markets differ in market tightness. In a market with low prices a seller can find a buyer very quickly and in a market with high prices it takes a long time to find a buyer. Low-quality sellers who are more eager to sell quickly self-select into the low price market while high-quality sellers are happy to wait longer in the high price market. One can relate our design questions to a search setting by studying the efficiency consequences of closing certain markets (for example, using a price ceiling). This would roughly correspond to closing the market after some time in our setting.

\section{The model with a known timing of shock}

As in the classic market for lemons, a potential seller owns one unit of an indivisible asset. When the seller holds the asset, it generates for him a revenue stream with net present value $c \in[0,1]$ that is private information of the seller. The seller's type, $c$, is drawn from a distribution $F(c)$, which is common knowledge, atomless and has a continuous, strictly positive density $f(c)$. At time $T \leq \infty$ the seller's type is publicly revealed. ${ }^{3}$

There is a competitive market of potential buyers. Each buyer values the asset at $v(c)$ which is strictly increasing, twice continuously differentiable, and satisfies $v(c)>c$ for all $c<1$ (i.e., common knowledge of gains from trade) and $v(1)=1$

\footnotetext{
2 See also Tirole (2012) and Philippon and Skreta (2012).

3 We could think of the public revelation of the banking stress tests as a possible example of this.
} 
(i.e., no gap on the top). These assumptions imply that in the static Akerlof (1970) problem some but not all types trade in equilibrium. 4

Time is $t \in[0, \infty]$ and we consider different market designs in which the market is opened at different moments in that interval. Note that the first time the market opens after private information is revealed, trade will take place immediately with probability 1 . So without loss we consider only $t \in[0, T]$ and assume that the market is always "opened" at $T$ (but see Section C.3 in the Appendix for the possibility of restricting trade also at $T$ and later).

Let $\Omega \subseteq[0, T]$ denote the set of times that the market is open (we assume that at the very least $\{0, T\} \subset \Omega$ ). We call $\Omega$ the timing design, motivated by a regulator or a market maker who can affect when the market is open (by its choice of $\Omega$ ).

There are many examples of possible timing designs; some examples are: (i) infrequent trading $\Omega_{I}=\{0, T\}$ (ii) continuous trading, $\Omega_{C}=[0, T]$, (iii) constant frequency of trading: $\Omega_{\Delta}=\{0, \Delta, 2 \Delta, \ldots, T\}$, and (iv) early closure design: $\Omega^{E C}=\{0\} \cup[\Delta, T]$.

Every time the market is open, there is a market price $p_{t}$ at which buyers are willing to trade, and the seller either accepts (which ends the game) or rejects it. If the price is rejected the game moves to the next time the market is open. If no trade takes place by time $T$, the type of the seller is revealed and the price in the market is $v(c)$, at which all seller types trade.

All players discount payoffs at a rate $r$, and we use $\delta=e^{-r \Delta}$ when convenient. If trade happens at time $t$ at a price $p_{t}$, the seller's payoff is

$$
\left(1-e^{-r t}\right) c+e^{-r t} p_{t}
$$

and the buyer's payoff is

$$
e^{-r t}\left(v(c)-p_{t}\right)
$$

\subsection{Equilibrium definition and examples}

There are many ways regulators or market makers may influence markets. For example, designers can tax or subsidize trade, or choose how much information to reveal (as in the literature on information design). In this paper we focus on the problem of a market designer who can choose timing design, while prices and trades are set by equilibrium forces that we define now. Despite timing design being a rather limited tool (as opposed to using arbitrary taxes and subsidies subject to a budget constraint), we show that under certain conditions timing design can be as efficient as an arbitrary balanced-budget policy. We also discuss how in this case a certain information policy can do as well.

Before defining equilibrium it is worth noting that the Skimming Property holds in this setting. That is, in any equilibrium, if a given type $c$ is willing to accept an offer $p_{t}$ at time $t$ then, all types $c^{\prime}<c$ would also accept this offer (had they not traded before). Since we know that the skimming property holds in this environment it is simpler to directly define the competitive equilibrium in terms of cutoffs.

A competitive equilibrium for a given timing design $\Omega$ is a pair of functions $\left\{p_{t}, k_{t}\right\}$ for $t \in \Omega /\{T\}$ where $p_{t}$ is the competitive market price at time $t$ and $k_{t}$ is the highest type of the seller that trades at time $t$. These functions must satisfy:

(1) Zero profit condition: $p_{t}=E\left[v(c) \mid c \in\left[k_{t-}, k_{t}\right]\right]$ where $k_{t-}$ is the cutoff type at the previous time the market is open before $t$ (with $k_{0-}=0$ for the first time the market is opened.) ${ }^{5}$

(2) Seller optimality: given the process of prices, and that prices at $T$ are $p_{T}(c)=v(c)$ (for every history of previous play), each seller type maximizes profits by trading according to the rule $k_{t}{ }^{6}$

(3) Market Clearing: in any period the market is open, the price is at least $p_{t} \geq v\left(k_{t-}\right)$.

Conditions (1) and (2) are straightforward. Condition (3) guarantees that there is no excess demand given the prices at times when the market is open but there is no trade. If the asset were offered at a price $p_{t}<v\left(k_{t-}\right)$ at time $t$, then, since the value of the good is at least $v\left(k_{t-}\right)$, there would be excess demand making those prices inconsistent with market clearing.

We assume that all market participants publicly observe all the trades. Hence, once a buyer obtains the asset, if he tries to put it back on the market, the market makes a correct inference about $c$ based on the history. Since we assume that all buyers have the same value of the asset, there would not be any profitable re-trading of the asset (after the initial seller transacts) and hence we ignore that possibility (however, see Remark 1).

To illustrate the model and the definition of equilibrium consider the timing design: $\Omega_{\Delta}=\{0, \Delta, 2 \Delta, \ldots\}($ with $T=\infty)$.

The equilibrium conditions at times the market is open are:

Zero profit condition:

$$
p_{t}=E\left[v(c) \mid c \in\left[k_{t-\Delta}, k_{t}\right]\right]
$$

\footnotetext{
4 Assuming $v(1)=1$ allows us not to worry about out-of-equilibrium beliefs after a history where all seller types are supposed to trade but the trade did not take place. We discuss this assumption further in Section C.4 in the Appendix.

5 In continuous time we use a convention $k_{t-}=\lim _{s \uparrow t} k_{s}, E\left[v(c) \mid c \in\left[k_{t-}, k_{t}\right]\right]=\lim _{s \uparrow t} E\left[v(c) \mid c \in\left[k_{s}, k_{t}\right]\right]$, and $v\left(k_{t-}\right)=\lim s \uparrow t\left(k_{s}\right)$. If $k_{t}=k_{t-}$ then the condition (combined with the market clearing condition 3 ) is $p_{t}=v\left(k_{t}\right)$.

6 Implicitly, for the equilibrium to exist we require that the price process is such that an optimal seller strategy exists.
} 


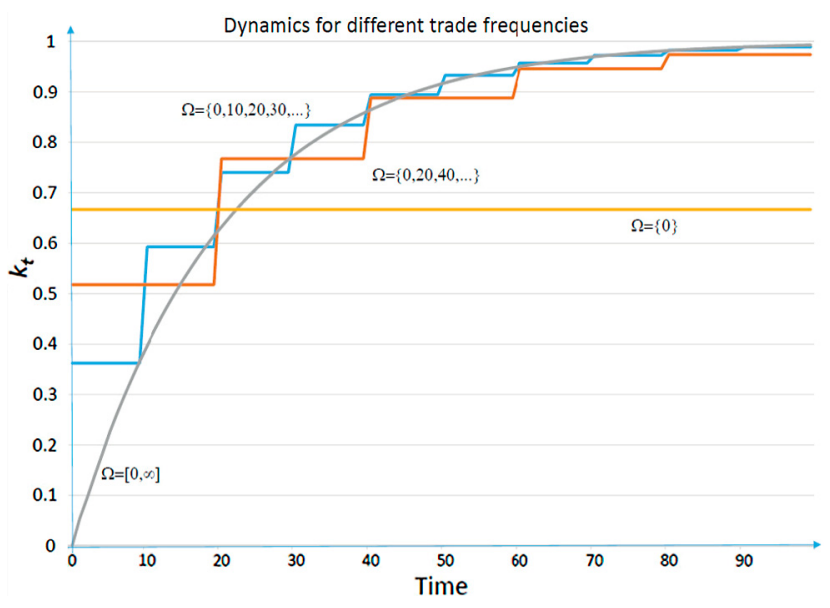

Fig. 1. Equilibrium dynamics for different trading frequencies.

\section{Seller optimality:}

$$
\left(p_{t}-k_{t}\right)=e^{-r \Delta}\left(p_{t+\Delta}-k_{t}\right) .
$$

The seller optimality conditions are the indifference conditions for each cutoff type trading at $t$, for every $t \in \Omega_{\Delta}$. They are necessary and sufficient for the seller optimality in case there is trade in every period.

From these two equations for given $\Delta$ we can derive a difference equation for equilibrium cutoffs and prices. ${ }^{7}$

An equilibrium for infrequent trading, $\Omega_{I}=\{0, T\}$, is characterized by just $\left\{p_{0}, k_{0}\right\}$ that satisfy:

$$
\begin{aligned}
& p_{0}=E\left[v(c) \mid c \in\left[0, k_{0}\right]\right] \text { and } \\
& \left(p_{0}-k_{0}\right)=e^{-r T}\left(v\left(k_{0}\right)-k_{0}\right),
\end{aligned}
$$

where the right-hand side of the second condition follows from the assumption that at $T$ seller's type becomes public and he sells for $p_{T}(c)=v(c)$.

Finally, in case of continuous trading, $\Omega_{C}=[0, T]$, the equilibrium is the unique solution to:

$$
\begin{aligned}
p_{t} & =v\left(k_{t}\right) \\
r\left(p_{t}-k_{t}\right) & =\frac{d p_{t}}{d t} \\
k_{0} & =0,
\end{aligned}
$$

where the first equation captures the zero-profit condition in case trade is atomless over time, the second equation is the indifference condition for the current cutoff type that implies global optimality, and the last equation is the boundary condition. ${ }^{8}$

Below we plot the path of cutoffs for different values of $\Delta$ for the case $c$ distributed uniformly over [0,1], $v(c)=$ $\frac{1+c}{2}, r=10 \%$, and $T=\infty$.

How does trading efficiency depend on $\Delta$ ? From Fig. 1, it is not obvious. As can be observed, there is generally a trade-off, with some types trading sooner as $\Delta$ increases and some types trading later. For our example we can compute the discounted realized gains from trade for different values of $\Delta$. Fig. 2 presents these results normalized by the full potential gains from trade.

Consider the two extreme cases: $\Omega_{I}=\{0\}$ and $\Omega_{C}=[0, \infty]$. Committing to only one opportunity to trade generates a big loss of surplus if there is no immediate trade. This clearly leaves a lot of unrealized gains from trade in our example: types between $2 / 3$ and 1 do not trade. However, it is this inefficiency upon disagreement that helps overcome the adverse selection problem and increases the amount of trade in the initial period (types $c \in[0,2 / 3]$ trade at time $t=0$ ). Continuous trading, on the other hand, does not provide many incentives to trade early since a seller suffers a negligible loss of surplus from delaying to the next instant. This leads to an equilibrium with smooth trading over time with only the lowest type

\footnotetext{
7 See Appendix B for a detailed derivation of the equilibrium for $\Omega_{I}=\{0\}$ and $\Omega_{C}=[0, \infty]$, and the proof of Theorem 2 for $\Omega_{\Delta}=\{0, \Delta, 2 \Delta, \ldots\}$ when $v(c)$ is linear and $F(c)=c$. These equations have a unique solution for the linear-uniform case, but in general there could be more than one solution and hence more than one equilibrium for a given $\Omega$. That is even true as $\Delta \rightarrow \infty$ so the model becomes static.

8 The intuition for the uniqueness of equilibrium for $\Omega_{C}$ is that if there was an atom of trade at some time $t$, then at $t+\varepsilon$ price would have to increase discontinuously and that would contradict optimality of the seller's strategy. See Fuchs and Skrzypacz (2015) for a detailed discussion.
} 


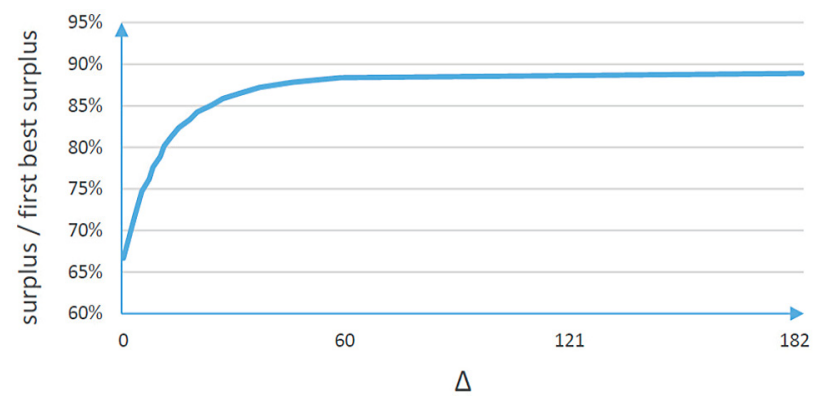

Fig. 2. Efficiency relative to first best for different frequencies of trade.

trading at $t=0$. While the screening of types via delay is costly, the advantage is that eventually (if $T$ is large enough) more types trade. In determining which trading environment is more efficient on average, one has to weight the cost of delaying trade with low types with the advantage of eventually trading with more types. In our example, the trade-off is always resolved in favor of trading less frequently, as illustrated in Fig. 2. When the market is continuously open, only $66 \%$ of the available surplus is attained, and when the market opens only once, $89 \%$ of the surplus is attained. In the next section we discuss the generality of this finding.

\section{Optimality of restricting trading opportunities with a known timing of shock}

Our examples above illustrated that restricting the timing of trade can be better than allowing continuous-time trading. In this section we characterize the optimal $\Omega$ (under some regularity conditions) and discuss other choices of $\Omega$. Our example so far compared continuous trading market with one-time trading and constant frequency of trading. There are many other natural possibilities when the timing of the shock is known. For example, the market could be opened at 0 , then closed for some time interval $\Delta$ and then be opened continuously. Or the market could start off being opened continuously and close for some $\Delta$ before $T$ (i.e., at $t=T-\Delta$ ).

\subsection{When infrequent trading is optimal}

The main result of this section is that under a relatively general set of conditions, the optimal design is to have infrequent trading $\Omega_{I}=\{0, T\}$. The result is that under the sufficient condition design $\Omega_{I}$ dominates $\Omega_{C}$ and any other $\Omega$ : closing the market at all intermediate periods is better than any other timing protocol (not just continuous trading).

A sufficient condition for our result is regularity defined as:

Definition 1. We say that the environment is regular if $\frac{f(c)}{F(c)} \frac{v(c)-c}{\left(1-e^{-r T}\right)+e^{-T T} v^{\prime}(c)}$ and $\frac{f(c)}{F(c)}(v(c)-c)$ are decreasing.

To understand the regularity condition, it is convenient to think of a static mechanism design problem but where the seller's reservation value is some increasing function $R(c)$. In our case, the outside option of type $c$ is to wait to trade at time $T$ at price $v(c)$, so $R(c)=\left(1-e^{-r T}\right) c+e^{-r T} v(c)$. With this notation, the first part of the regularity condition can be written as:

$$
\frac{v(c)-R(c)}{R^{\prime}(c) \frac{F(c)}{f(c)}} \text { is decreasing. }
$$

As we explain below, the numerator captures the gains from trade with type $c$ at time 0 , and the denominator measures the information rents effect of trading with this type. When this ratio is decreasing, the marginal gains from trade per unit cost necessary to induce higher types to trade are decreasing in $c$. This helps us prove that the optimal market design is such that some types trade at $t=0$ for sure and all other types wait to trade at $T$. The option to wait till $T$ has a special role not present in standard static mechanism design: if the mechanism calls for the seller to not trade before $T$, we assume that at that point the seller can capture the full surplus by selling his asset in the competitive market at price $v(c)$. Hence his outside option is a function of the buyer's value, $v(c)$ (and his information rent is a function of $v^{\prime}(c)$ ).

Theorem 1. If the environment is regular then infrequent trading, $\Omega_{I}=\{0, T\}$, generates higher expected gains from trade than any other market design. ${ }^{9}$

\footnotetext{
${ }^{9}$ Omitted proofs can be found in Appendix A.
} 
We establish this theorem by proving an even stronger result. Suppose that a market designer could design an arbitrary direct revelation mechanism in which the seller would report $c$, and the buyers would obtain the good at some price, subject to the following constraints: (i) the buyers do not pay more than the expected value of the asset that they receive; (ii) the market designer has on average a balanced budget (but can cross-subsidize types); (iii) the seller finds it optimal to report $c$ truthfully, and his participation constraint is satisfied (i.e., the seller payoff is at least $R(c)$ that corresponds to holding the asset till $T$ ); (iv) if the mechanism does not call the seller to sell before $T$, the seller obtains payoff $R(c)$.

Such a direct revelation mechanism describes, as a function of reported type, three objects: the probability that the seller holds the asset till $T, y(c)$; the probability distribution over selling times in $[0, T)$ conditional on selling before $T, G_{t}(c)$; and the expected net present value of the payment the seller receives, $P(c)$. Compared to the timing design alone, this more general class of mechanisms gives the market designer the flexibility of taxing and subsidizing trade at different times and potentially cross-subsidizing trades of different types.

In the proof, we show that, under the regularity conditions, the solution to the relaxed problem is that types below a threshold trade immediately and types above the threshold wait till $T$, with no trade in the middle. That solution to the relaxed problem can be implemented by the $\Omega_{I}$ design. (If design $\Omega_{I}$ leads to multiple equilibria, our theorem applies to the one in which the threshold $k_{0}$ is the highest across all equilibria). So indeed, design $\Omega_{I}$ maximizes total surplus even in this much broader class of mechanisms (i.e., not only over all feasible $\left.\Omega^{\prime} s\right)^{10}$

A detailed proof of this result is in the Appendix. To illustrate what is new about this mechanism design problem (and why one of the regularity conditions involves $v^{\prime}(c)$ when $\left.T<\infty\right)$, consider a simpler mechanism design problem in which the designer tries to maximize expected gains from trade by choosing a (direct revelation) mechanism that determines for each type the probability of trade at time $0, z(c)$, and expected payment conditional on trade, $P(c)$. We require the mechanism to be incentive compatible, individually rational and budget-balanced on average. If type $c$ does not trade at $t=0$, it obtains outside option payoff $R$ (c) (in case the outside option is to wait till $T$ and trade at $v(c)$, then as we stated above, $\left.R(c)=\left(1-e^{-r T}\right) c+e^{-r T} v(c)\right)$.

The objective of the mechanism designer is to maximize

$$
\int_{0}^{1} z(c)(v(c)-R(c)) f(c) d c .
$$

The budget-balance condition is

$$
\int_{0}^{1} z(c)(v(c)-P(c)) f(c) d c \geq 0
$$

since the most the mechanism designer can charge the buyers is $v(c)$ and he has to pay $P(c)$ to the seller.

The seller's expected net surplus from trade is:

$$
\begin{aligned}
W(c) & =z(c)(P(c)-R(c)) \\
& =\max _{c^{\prime}} z\left(c^{\prime}\right)\left(P\left(c^{\prime}\right)-R(c)\right) .
\end{aligned}
$$

That allows us to state the budget constraint as

$$
\int_{0}^{1}[z(c)(v(c)-R(c))-W(c)] f(c) d c \geq 0 .
$$

Further, we use the envelope formula to express the last term as

$$
\int_{0}^{1} W(c) f(c) d c=\int z(c) R^{\prime}(c) \frac{F(c)}{f(c)} f(c) d c
$$

which captures the expected information rents of the seller.

\footnotetext{
10 For $T=\infty$, this is a problem analyzed in Samuelson (1984) and in Fuchs and Skrzypacz (2015). The novelty in Theorem 1 is that it allows for a finite $T$ (and for that reason it requires new and different regularity conditions). From a practical perspective, solving the model with temporary information asymmetry allows us to think of situations such as stress tests or other forms or regulatory approval.
} 
Putting it together, the mechanism designer solves the problem

$$
\begin{aligned}
& \max _{z(c) \in[0,1]} \int_{0}^{1} z(c)(v(c)-R(c)) f(c) d c \\
& \text { s.t. } \\
& 0 \leq \int_{0}^{1} z(c)\left(v(c)-R(c)-R^{\prime}(c) \frac{F(c)}{f(c)}\right) f(c) d c \\
& z(c) \text { is weakly decreasing }
\end{aligned}
$$

where the monotonicity of $z(c)$ is necessary for the mechanism to be incentive compatible. For now, focus on the relaxed problem ignoring that monotonicity constraint on $z(c)$. For $c$ such that the integrand in the budget constraint is positive, it is optimal to set $z(c)=1$ : this relaxes the budget constraint and improves the objective function. The regularity condition guarantees that $\left(v(c)-R(c)-R^{\prime}(c) \frac{F(c)}{f(c)}\right)$ changes sign from positive to negative only once. Now consider $c^{\prime} s$ such that this expression is negative. Setting $z(c)=1$ tightens the budget constraint but improves the objective. The optimal way to choose which $c$ should trade at $t=0$ is to compare the bang-for-the-buck of different $c$ 's. That is, what is the contribution of their trade to the objective function per unit cost in the budget constraint. The bang-for-the-buck ratio varies one-to-one with:

$$
\frac{v(c)-R(c)}{R^{\prime}(c) \frac{F(c)}{f(c)}} \text {. }
$$

If that ratio is decreasing (as assumed in the regularity condition), the optimal solution is bang-bang: have low types trade for sure at $t=0$ and all higher types wait till $T$, with the threshold $c^{*}$ determined by the budget constraint

$$
\int_{0}^{c^{*}}\left(v(c)-R(c)-R^{\prime}(c) \frac{F(c)}{f(c)}\right) f(c) d c=0 .
$$

A way to implement this mechanism is to have a price equal to $E\left[v(c) \mid c \leq c^{*}\right]$ at $t=0$, and that is consistent with an equilibrium with design $\Omega_{I}=\{0, T\}$.

The proof of the theorem is a bit more complex than this simple intuition since we allow the planner to have some types trade with positive probability at $t \in(0, T)$. This possibility accounts for the second regularity condition that we need to establish the result. In the proof we define $x(c) \equiv \int_{0}^{T} e^{-r t} d G_{t}(c)$ as the expected discount factor at the time of trade before $T$ (seller's incentives depend on $G_{t}(c)$ only via $x(c)$ ), and we use $\delta=e^{-r T}$.

With this notation, the seller chooses two allocation functions, $y(c) \in[0,1]$ which is the probability that type $c$ has to wait till $T$, and $x(c) \in(\delta, 1]$ which is the expected discount if type $c$ is told to trade before $T$, and a payment function $P(c)$. These are chosen to maximize expected gains from trade:

$$
\max _{x, y} \int_{0}^{1}(1-y(c))(x(c) v(c)+(1-x(c)) c-R(c)) f(c) d c .
$$

Seller's expected net surplus from is:

$$
\begin{aligned}
W(c) & =(1-y(c))[P(c)+(1-x(c)) c-R(c)] \\
& =\max _{c^{\prime}}\left(1-y\left(c^{\prime}\right)\right)\left[P\left(c^{\prime}\right)+\left(1-x\left(c^{\prime}\right)\right) c-R(c)\right] .
\end{aligned}
$$

By the envelope theorem we have:

$$
W^{\prime}(c)=(1-y(c))\left(1-x(c)-R^{\prime}(c)\right) .
$$

The rest of the proof is similar to our discussion above when only trade at $t \in\{0, T\}$ is allowed. The novelty of this mechanism design problem with finite horizon is that there are two allocation instruments, $x$, and $y$ that enter $W^{\prime}(c)$ differently (one multiplies $R^{\prime}(c)$ and the other not, because only $y(c)$ is related to waiting till $T$ ). The two regularity conditions are sufficient for the partial derivatives (with respect to the two instruments) of the Lagrangian to cross zero only once (as $c$ varies) and hence to yield a bang-bang characterization of the optimum: $x(c)=1-y(c)=1$ for types below a threshold, while $y(c)=1$ for types above that threshold (and that allocation is the equilibrium outcome with $\Omega_{I}$ ).

If the solution to the relaxed problem does not have the property that all trade takes place only at $t=0$ or $t=T$, then it involves the cross-subsidization of the buyers, and the allocation of the relaxed mechanism cannot be implemented as a competitive equilibrium without the use of taxes and subsidies. It is an open question how to solve for the optimal $\Omega$ if the solution to the relaxed problem calls for trade in more than one period before $T$. 
Commitment to infrequent trading Although it might be optimal to have just a unique trading opportunity, ex-post (i.e., after time 0 ) there would be an incentive to trade again instead of waiting till $T$. Hence an important practical question is if $\Omega_{I}$ can be implemented. With no commitment, no credible way of stopping parties from trading, the equilibrium would be the one with continuous trading opportunities that we know is inefficient (at least when the regularity conditions hold). From a market design perspective this paper highlights that it is valuable to be able to restrict trading opportunities credibly.

We propose anonymity and secrecy of market transactions as a possible tool a market designer could use to achieve an effective market closure even if the market cannot be physically closed:

Remark 1. One way to implement $\Omega_{I}=\{0, T\}$ in practice may be via an Extreme Anonymity of the market. That is, a market design in which transactions and identity of traders are unobservable (for example, because goods are transacted secretly by a market-maker). In our model we have assumed that the initial seller of the asset can be told apart in the market from buyers who later become secondary sellers. However, if the trades are completely anonymous, even if $\Omega \neq\{0, T\}$, the equilibrium outcome would coincide with the outcome for $\Omega_{I}$.

Formally establishing this result would require modeling buyers as strategic players which would require introducing a lot of notation. Yet, the main idea is simple: Extreme Anonymity exacerbates the adverse selection problem after time 0 . As long as the players' value for the asset does not change, a buyer at time $t>0$ would be facing a worse adverse selection problem since it would potentially be trading with sellers with whom there are no gains from trade (i.e., those that bought the asset from the original seller at an earlier time). As a result, under Extreme Anonymity, prices can never go up: otherwise buyers who purchased the good earlier would resell them at the later markets, and late buyers would lose money. More concretely, suppose there where some time $t>0$ with $p_{t}>p_{0}$. Who would sell at this price? Buyers who bought the asset at $t=0$ and found out that $v(c)<p_{t}$, and sellers whose type is below $p_{t}$ and who didn't expect other opportunities to trade. Note that this pool of types is strictly worse than what we would obtain if $p_{t}$ had been offered in the first period. Since it was not profitable to offer $p_{t}$ at time zero it must lead to strict losses when offered at $t>0$.

Such extreme anonymity may not be feasible in some markets (for example, IPO's), or not practical for reasons outside the model. Yet, it may be feasible in some situations. For example, a government as a part of an intervention aimed at improving the efficiency of the market may create a trading platform in which it would act as a broker who anonymizes trades and traders.

\subsection{Beyond the regular case: temporary closures}

What if the environment is not regular? We do not have a complete characterization of this case, but can provide some partial answers.

First, some conditions are indeed necessary for $\Omega_{I}$ to be optimal as this result illustrates:

Proposition 1. In general, the ranking of the efficiency attained with continuous trading and infrequent trading $\left(\Omega_{C} v s . \Omega_{I}\right)$ is ambiguous.

The example used in the proof of this proposition illustrates what could make the continuous trading market superior to the infrequent one. We need a large mass at the bottom of the distribution, so that the infrequent trading market gets "stuck" with only these types trading. Under continuous trading these types trade quickly, so the delay costs for these types are small. Additionally, we need some mass of higher types that would be reached in the continuous trading market after some time, generating additional surplus. Alternatively, one can construct examples in which the gains from trade are small for low types and large for intermediate types, so that some delay cost at the beginning is more than compensated by the increased overall probability of trade.

This result highlights the contrast with respect to the model of Spence (1973) in which it is always true that restricting all signaling opportunities is optimal. The difference is that in our setting there is no pooling offer that would simultaneously satisfy the break-even condition and have all types trading. This follows since the reservation value of the highest type is 1 and $E[v(c) \mid c \in[0,1]]<1$.

Second, we can show quite generally that $\Omega_{C}$ is not optimal for any $F$ or $v$. In particular, consider the design $\Omega^{E C} \equiv$ $\{0\} \cup[\Delta, T]$ : trade is allowed at $t=0$, then the market is closed till $\Delta>0$ and then it is opened continuously till $T$. We call this design "early closure". We show that one can always find $\Delta>0$ that improves upon continuous trading:

Result 1. Allowing for continuous trading is never optimal. For every $r, T, F(c)$, and $v(c)$, there exists $\Delta>0$ such that with the early closure market design $\Omega^{E C}=\{0\} \cup[\Delta, T]$ all types are weakly better off with $\Omega^{E C}$ relative to the continuous trading design $\Omega_{C}=[0, T]$ and some are strictly better off.

The proof of this result follows from the proof of Lemma 2 in FS so we do not repeat it here. The key is that for small $\Delta$ with $\Omega^{E C}$ there is more trade overall and all types that trade do it sooner. So, the social surplus is higher type-by-type. To see this, let $k_{\Delta}^{E C}$ be the highest type that trades at $t=0$ when the design is $\Omega^{E C}$ and let $k_{\Delta}^{C}$ the equilibrium cutoff at time $\Delta$ in design $\Omega_{C}$. We show that for small $\Delta, k_{\Delta}^{C}<k_{\Delta}^{E C}$. Since with $\Omega^{E C}$ once the market re-opens at $\Delta$ the equilibrium is 


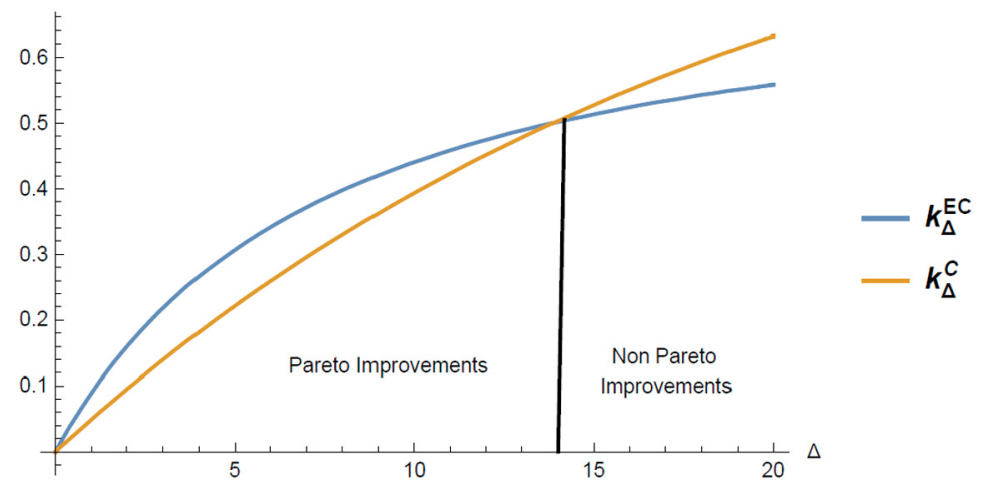

Fig. 3. Trade at time zero with early closure of size $\Delta$ vs. amount of trade by time $\Delta$ with continuous trading.

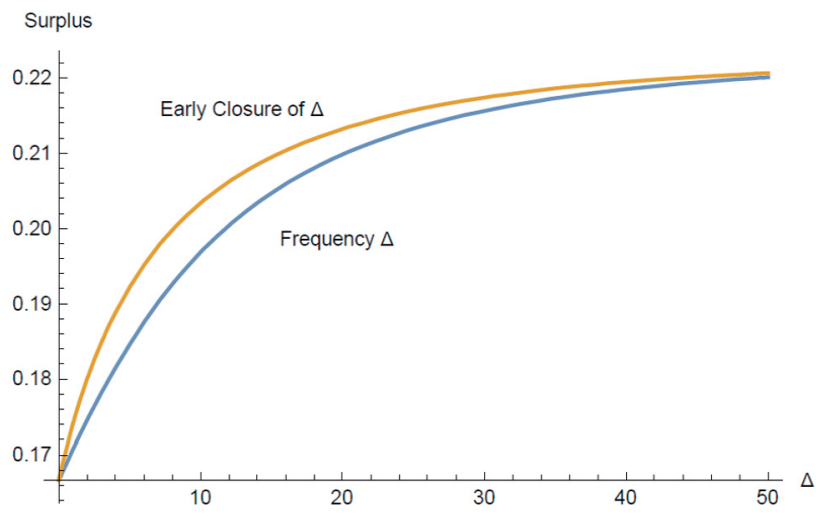

Fig. 4. Surplus in Early Closure vs. Discrete Grid of Trading Times.

the same as in case of $\Omega_{C}$ but with the different boundary condition (i.e., the lowest type that trades at $t=\Delta$ ), the claim follows. In the example in Fig. 3, all early closures with $\Delta<14$ have $k_{\Delta}^{C}<k_{\Delta}^{E C}$ and thus lead to a Pareto improvement. In Fig. 3 we can also observe that as $\Delta \rightarrow 0$ both $k_{\Delta}^{E C}$ and $k_{\Delta}^{C}$ converge to 0 but that the slope at the origin is higher for $k_{\Delta}^{E C}$ than for $k_{\Delta}^{C}$. Indeed, this is a general feature and we can show that $\lim _{\Delta \rightarrow 0} \frac{\partial k_{\Delta}^{E C}}{\partial \Delta}=2 \lim _{\Delta \rightarrow 0} \frac{\partial k_{\Delta}^{C}}{\partial \Delta}$.

To understand the economics behind this result, note first that when the market is closed in $(0, \Delta)$ even if the price at 0 does not change, some types that were planning to trade in $(0, \Delta)$ now prefer to trade at 0 rather than at $\Delta$. This of course implies that the pool of sellers at $t=0$ is strictly better and thus $p_{0}$ must increase. The increase in $p_{0}$ leads to more types wanting to trade at $t=0$ and so on. The effect on $p_{0}$ of adding additional types is reduced as the pool gets larger so this virtuous circle eventually comes to a stop. Thus, while true for small $\Delta$, the result does not hold for arbitrary $\Delta$ as seen in Fig. 3. ${ }^{11}$

It is perhaps natural to expect that if a first closure of size $\Delta$ followed by continuous trading, $\Omega^{E C}=\{0\} \cup[\Delta, T]$, leads to an improvement, a grid of trades at intervals $\Delta, \Omega_{\Delta}=\{0, \Delta, 2 \Delta, \ldots\}$, would lead to further improvements. However, it turns out that this is generally not the case, as we illustrate in Fig. 4 .

The main reason $\Omega_{\Delta}$ yields a lower total surplus than $\Omega^{E C}$ is that the size of the first atom is smaller in the former timing design. Suppose we started with $\Omega^{E C}$ and eliminated the opportunities to trade in $(\Delta, 2 \Delta)$. Now, some types that were trading in $(\Delta, 2 \Delta)$ would choose to trade at $\Delta$, which would lead to an increase of $p_{\Delta}$. But now, some of the types that were trading at $t=0$ would prefer to delay their trade to time $\Delta$. That would reduce $p_{0}$ and reinforce the incentives for some types to delay their trade to time $\Delta .^{12}$ Thus, in equilibrium we have less trade at $t=0$ with $\Omega_{\Delta}$ than with $\Omega^{E C}$. It seems natural to argue that there must be some gain from types originally trading in $(\Delta, 2 \Delta)$ now trading at $\Delta$. But this gain is offset by the loss that arises from the higher types that used to trade in $(\Delta, 2 \Delta)$ now trading at $2 \Delta$ instead. We explore this intuition further in the appendix (Section C.1) when we analyze $\Omega$ with one closure before $T$.

\footnotetext{
11 The fact that $\lim _{\Delta \rightarrow 0} \frac{\partial k_{\Delta}^{E C}}{\partial \Delta}=2 \lim _{\Delta \rightarrow 0} \frac{\partial k_{\Delta}^{C}}{\partial \Delta}$ follows from $f(c)$ and $v(c)$ being positive and continuous. For small $\Delta$ they are thus locally approximately linear-uniform (as in the example plotted in Fig. 3), hence when types start pooling at $t=0$, in equilibrium prices grow at half the speed of $v\left(k_{\Delta}^{C}\right)$, which leads to $k_{\Delta}^{E C}$ being approximately twice as high as $k_{\Delta}^{C}$.

12 Indeed, this partially undoes the virtuous circle we described above and the slope of the welfare at the origin with $\Omega_{\Delta}$ can be shown to be half of that with $\Omega^{E C}$ in the linear-uniform case.
} 


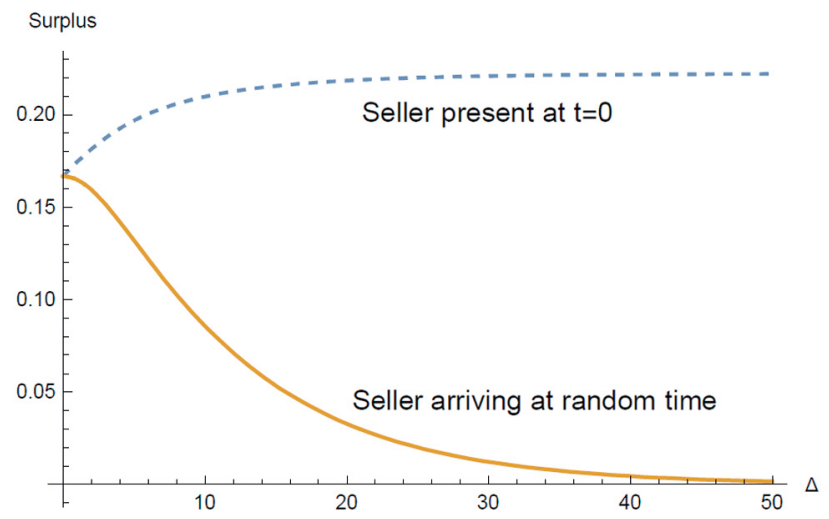

Fig. 5. The differential effects of frequency of trade depending on the seller being present or randomly arriving.

\section{Optimal frequency of trade with stochastic arrival of shocks}

The analysis thus far assumes that the seller is present at time zero or that opportunities to trade can be tailored to individual sellers. This abstraction works well when thinking of clear distress episodes or formal bankruptcy proceedings but not the day to day workings of a financial exchange. In such settings it is natural to assume instead that traders arrive randomly over time and any restriction to trade must be uniformly applied to all those present, regardless of when they arrived. We consider this case next. To do so, we assume the sellers arrive at a Poisson rate $\lambda$ and that the market timing cannot be tailored to the realized time of the arrival. In this case, it is less natural to think of a finite horizon. Thus, we limit our analysis to the case $T=\infty$. For prices, we still assume that the market observes when the seller starts looking for a buyer. That is, the arrival time of the seller is observable by the market but not by the market designer. Since there is no sense of a time zero, it is also natural to consider only the set of equally spaced trading times $\Omega_{\Delta}=\{0, \Delta, 2 \Delta, 3 \Delta, \ldots\}$ (where $\Omega_{C}$ corresponds to the limiting case $\Delta \rightarrow 0$ ). Thus, the question of interest is what is the optimal $\Delta$.

Equilibrium properties and welfare for a given $\Delta$.

First, let's consider the equilibrium objects at times the market is open conditional on the seller having arrived (recall that the arrival is observable by the market):

For prices:

$$
p_{t}=E\left[v(c) \mid c \in\left[k_{t-\Delta}, k_{t}\right]\right]
$$

For cutoffs:

$$
\left(p_{t}-k_{t}\right)=e^{-r \Delta}\left(p_{t+\Delta}-k_{t}\right)
$$

Combining both equilibrium conditions we get:

$$
E\left[v(c) \mid c \in\left[k_{t-\Delta}, k_{t}\right]\right]-k_{t}=e^{-r \Delta}\left(E\left[v(c) \mid c \in\left[k_{t}, k_{t+\Delta}\right]\right]-k_{t}\right)
$$

In general, this second-order difference equation is quite hard to work with. However, in the linear-uniform case, i.e., $v(c)=\alpha c+(1-\alpha)$ and $F(c)=c$, we get a tractable second-order linear difference equation for $k_{t}$. We can solve this equation (see the Appendix) and calculate the expected welfare for different values of $\Delta$, which we denote by $S\left(\Omega_{\Delta}\right)$. Importantly, $S\left(\Omega_{\Delta}\right)$ accounts for the cost of waiting for the market to reopen if the seller arrives at a time the market is closed.

As we showed in Theorem 1, since the linear-uniform case satisfies the regularity condition, it is optimal in to set $\Delta=\infty$ if the seller arrives at $t=0$. Albeit, now there is the additional consideration that the trader might arrive when the market is closed, in which event he would have to wait for the market to reopen. As a result, since the probability that the trader arrives at $t=0$ is zero, $\lim _{\Delta \rightarrow \infty} S\left(\Omega_{\Delta}\right)=0$. Thus $\Delta=\infty$ can not be optimal. On the other hand, in the other limit $(\Delta \rightarrow 0)$, the market is always open, so once the seller arrives it can start trading immediately. For that design, the expected gains from trade from the time the seller arrives are the same whether the seller is known to arrive at $t=0$ or to arrive randomly.

Fig. 5 plots the expected welfare conditional on the seller being present at $t=0$ (dashed) versus $S$ ( $\Omega_{\Delta}$ ) (solid) (i.e., when the seller arrives with intensity $\lambda=1$ and $r=10 \%$ ) for the linear-uniform case with $\alpha=\frac{1}{2}$.

As this example clearly shows, once we take into consideration the fact that traders might not be present at $t=0$ our conclusions change dramatically: now, the expected surplus is increasing in the frequency of trade. This suggests that while we might want to allow anonymous financial markets to trade continuously, when deciding bankruptcy proceedings in which there is a clear start date for the liquidation of assets, restricted trading opportunities might be more beneficial. Theorem 2 formalizes this finding. 
Theorem 2. For $v(c)=\alpha c+(1-\alpha)$ and $F(c)=c$, if sellers arrive randomly over time with Poisson intensity $\lambda$ then for any $\Delta>0$, $S\left(\Omega_{\Delta}\right)<S\left(\Omega_{C}\right)$. Moreover, $S\left(\Omega_{\Delta}\right)$ is decreasing in $\Delta$ for all $\Delta>0$.

\section{Conclusions}

In this paper we analyzed a dynamic market with asymmetric information. The main observation is that there is a big difference between the case in which there is a clear shock that generates the gains from trade and normal times in which the gains from trade arise stochastically over time. In the former case, it is typically optimal to restrict trades to only take place once and as early as possible. In the latter case, we have shown that it is best to allow trades to take place continuously, so that as soon as there are gains from trade, the seller can start trying to sell its asset.

We have shown that increasing the opportunities to trade makes the adverse selection problem worse since the common knowledge of gains from trading today vis a vis the next opportunity to trade shrink as that next opportunity becomes more immediate. Despite this effect, when opportunities to trade arise randomly, the cost of waiting till the next trading time when the seller arrives in between trading times is more severe than these benefits.

Although the model is stylized, the policy implications of our findings would be that normal times call for a laissez-faire approach, but when there is a big shock, such as the recent financial crisis, intervention and trade restrictions can increase welfare. The latter implication also applies to situations such as bankruptcy proceedings in which trading restrictions can be targeted to a particular firm. In this case, after the management decides on which assets to sell in the re-organization, it would be optimal to have just one organized auction in which these assets are sold.

Many open questions remain. First, if we think of a rich market setting with many sellers and buyers that both arrive over time, restricting opportunities to trade would have additional effects from the potential change on the demand side. This might be of little consequence when the size of the assets being sold is "small" relative to the market (our implicit assumption in this model) but could have an important effect when the size of the assets being sold is "large," where liquidity would become of first-order importance. On a more technical direction, it is an open question as to how to compute the optimal $\Omega$ in Theorem 1 if our regularity conditions do not hold. Lastly, it would be interesting to enrich the market micro-structure aspects of the model to study in more detail the benefits of allowing for high-frequency trading in an environment where there could be front-running or stale quotes.

One could also think of adding more dimensions of heterogeneity. For example, as pointed out recently by Roy (2014), a dynamic market can suffer from an additional inefficiency if buyers are heterogeneous. High valuation buyers are more eager to trade sooner and they may be the efficient buyers of the high-quality goods. Incorporating these considerations into our design questions may introduce new trade-offs.

When regulating or designing markets, regulators or designers may not have at their disposal the full set of tools of Mechanism Design. But in dynamic environments, Timing Design may still be available and lead to important welfare effects.

\section{Appendix A. Omitted proofs}

Proof of Theorem 1. We use mechanism design to establish the result. Consider the following relaxed problem. There is a mechanism designer who chooses a direct revelation mechanism that maps reports of the seller to (i) a probability distribution over times he trades, and (ii) transfers from the buyers to the mechanism designer, and (iii) transfers from the designer to the seller. The constraints on the mechanism are: incentive compatibility for the seller (to report truthfully); individual rationality for the seller and buyers (the seller prefers to participate in the mechanism rather than wait till $T$ and get $v(c)$, and the buyers do not lose money on average); and that the mechanism designer does not lose money on average. Additionally, we require that the highest type, $c=1$, does not trade until $T$ (as in any equilibrium he does not).

For every game with a fixed $\Omega$, the equilibrium outcome can be replicated by such a mechanism, but not necessarily vice versa - if the mechanism calls for the designer cross-subsidizing buyers across periods, it cannot be replicated by a competitive equilibrium.

Within this class of direct mechanisms we characterize one that maximizes ex-ante expected gains from trade. We then show that if the environment is regular, infrequent trading replicates the outcome of the best mechanism and hence any other timing design generates lower expected gains from trade.

A general direct revelation mechanism can be described by 3 functions $y(c), x(c)$ and $P(c)$, where $y(c)$ is the probability that the seller will not trade before information is released, $x(c)$ is the discounted probability of trade over all possible trading times and $P(c)$ is the transfer received by the seller conditional on trading before information is released. ${ }^{13}$ Note that $y(c) \in[0,1]$ but $x(c) \in[\delta, 1]$ where $\delta=e^{-r T}$.

13 Letting $G_{t}(c)$ denote for a given type the distribution function over the times of trade:

$$
\chi(c) \equiv \int_{0}^{T} e^{-r t} d G_{t}(c) .
$$


The seller's value function in the mechanism is:

$$
\begin{aligned}
U(c) & =y(c)[(1-\delta) c+\delta v(c)]+(1-y(c))[P(c)+(1-x(c)) c] \\
& =\max _{c^{\prime}} y\left(c^{\prime}\right)[(1-\delta) c+\delta v(c)]+\left(1-y\left(c^{\prime}\right)\right)\left[P\left(c^{\prime}\right)+\left(1-x\left(c^{\prime}\right)\right) c\right]
\end{aligned}
$$

Using the envelope theorem ${ }^{14}$ :

$$
\begin{aligned}
U^{\prime}(c) & =y(c)\left[(1-\delta)+\delta v^{\prime}(c)\right]+(1-y(c))(1-x(c)) \\
& =\delta y(c)\left(v^{\prime}(c)-1\right)+1-x(c)(1-y(c))
\end{aligned}
$$

Let $V(c)=\delta v(c)+(1-\delta) c$ be the surplus from not trading until $T$, so:

$$
\begin{aligned}
U^{\prime}(c)-V^{\prime}(c) & =\delta y(c)\left(v^{\prime}(c)-1\right)+1-x(c)(1-y(c))-\left(\delta v^{\prime}(c)+(1-\delta)\right) \\
& =(1-y(c))\left(-x(c)-\delta\left(v^{\prime}(c)-1\right)\right)
\end{aligned}
$$

As a result, we can write the expected seller's gains from trade as a function of the allocations $x(c)$ and $y(c)$ only:

$$
\begin{aligned}
S & =\int_{0}^{1}(U(c)-V(c)) f(c) d c \\
& =\left.(U(c)-V(c)) F(c)\right|_{c=0} ^{c=1}-\int_{0}^{1}\left(U^{\prime}(c)-V^{\prime}(c)\right) F(c) d c \\
& =\int_{0}^{1}(1-y(c))\left[x(c)-\delta\left(1-V^{\prime}(c)\right)\right] F(c) d c
\end{aligned}
$$

Clearly, the mechanism designer will leave the buyers with no surplus (since he could use it to increase efficiency of trade), and so maximizing $S$ is the designer's objective. That also means that the no-losses-on-average constraint simplifies to:

$$
\int_{0}^{1}(1-y(c))(x(c) v(c)-P(c)) f(c) d c \geq 0
$$

From the expression for $U(c)$ we have

$$
\begin{array}{r}
U(c)-y(c)[(1-\delta) c+\delta v(c)]-(1-y(c))(1-x(c)) c=(1-y(c)) P(c) \\
U(c)-V(c)+(1-y(c))(\delta(v(c)-c)+x(c) c)=(1-y(c)) P(c)
\end{array}
$$

So the constraint can be re-written as a function of the allocations alone (where the last term expands as in (2)):

$$
\int_{0}^{1}(1-y(c))(x(c)-\delta)(v(c)-c) f(c) d c-\int_{0}^{1}(U(c)-V(c)) f(c) d c \geq 0
$$

We now optimize (2) subject to (3), ignoring necessary monotonicity constraints on $x(c)$ and $y(c)$ that assure that reporting $c$ truthfully is incentive compatible (we check later that they are satisfied in the solution).

The derivatives of the Lagrangian with respect to $x(c)$ and $y(c)$ are:

$$
\begin{aligned}
L_{x}(c) & =(1-y(c))[F(c)+\Lambda((v(c)-c) f(c)-F(c))] \\
-L_{y}(c) & =\left(x(c)-\delta\left(1-v^{\prime}(c)\right)\right) F(c)+\Lambda\left[(x(c)-\delta)(v(c)-c) f(c)-\left(x(c)-\delta\left(1-v^{\prime}(c)\right)\right) F(c)\right]
\end{aligned}
$$

where $\Lambda>0$ is the Lagrange multiplier.

Consider $L_{x}(c)$ first. Note that $[F(c)+\Lambda((v(c)-c) f(c)-F(c))]$ is positive for $c=0$. Suppose $\frac{f(c)}{F(c)}(v(c)-c)$ is decreasing (which is part of the regularity assumption). Let $c^{*}$ be the unique solution to $1-\frac{f(c)}{F(c)}(v(c)-c)=\frac{1}{\Lambda}$ (if it exists, otherwise, let $\left.c^{*}=1\right)$. If $c^{*}<1$ then the second term in $L_{x}(c)$ changes sign once at $c^{*}$. An optimal $x(c)$ is therefore:

14 This derivative exists almost everywhere and hence we can use the integral-form of the envelope formula, (2). 


$$
x(c)=\left\{\begin{array}{l}
1 \text { if } c \leq c^{*} \\
\delta \text { if } c>c^{*}
\end{array}\right.
$$

Now consider $-L_{y}(c)$. For all $c \leq c^{*}$, using the optimal $x(c)$, it simplifies to:

$$
-L_{y}(c)=\left(1-\delta+\delta v^{\prime}(c)\right) F(c)+\Lambda\left[(1-\delta)(v(c)-c) f(c)-\left(1-\delta+\delta v^{\prime}(c)\right) F(c)\right] \text { for } x(c)=1
$$

If $\frac{f(c)}{F(c)} \frac{v(c)-c}{1+\frac{\delta}{(1-\delta)} v^{\prime}(c)}$ is decreasing in $c$, (which is part of the regularity assumption), $L_{y}(c)$ changes sign once in this range. It is negative for $c \leq c^{* *}$ and positive for $c>c^{* *}$, where $c^{* *} \leq c^{*}$ is a solution to $(1-\delta) \frac{f(c)}{F(c)}(v(c)-c)=$ $\left(1-\frac{1}{\Lambda}\right)\left(1+\frac{\delta}{(1-\delta)} v^{\prime}(c)\right)$. Therefore the optimal $y(c)$ in this range is

$$
y(c)=\left\{\begin{array}{l}
0 \text { if } c \leq c^{* *} \\
1 \text { if } c>c^{* *}
\end{array}\right.
$$

For $c>c^{*}$, using the optimal $x(c)$, the derivative $L_{y}(c)$ simplifies to

$$
L_{y}(c)=-(1-\Lambda) \delta v^{\prime}(c) F(c) \text { for } x(c)=\delta
$$

If $\Lambda>1$, this is positive and the optimal $y(c)$ is equal to 1 . If $\Lambda \leq 1, c^{*}=1$ and hence this case would be empty.

That finishes the description of the optimal allocations in the relaxed problem: there exists a $c^{*}$ such that types below $c^{*}$ trade immediately and types above it wait till after information is revealed at $T$. The higher the $c^{*}$ the higher the gains from trade. The largest $c^{*}$ that satisfies the constraint (3) is the largest solution of:

$$
E\left[v(c) \mid c \leq c^{*}\right]=(1-\delta) c^{*}+\delta v\left(c^{*}\right)
$$

since the LHS is the IR constraint of the buyers and the RHS is the IR constraint of the $c^{*}$ seller. This is also the equilibrium condition in a market with design $\Omega_{I}=\{0, T\}$, so that equilibrium implements the solution to the relaxed problem.

Proof of Proposition 1. Consider a distribution that approximates the following: with probability $\varepsilon, c$ is drawn uniformly on $[0,1]$; with probability $\alpha(1-\varepsilon)$ it is uniform on $[0, \varepsilon]$; and with probability $(1-\alpha)(1-\varepsilon)$ it is uniform on $\left[c_{1}, c_{1}+\varepsilon\right]$ for some $c_{1}>v(0)$. In other words, the mass is concentrated around 0 and $c_{1}$. Let $v(c)=\frac{1+c}{2}$ as in our example.

For small $\varepsilon$ there exists $\alpha<1$ such that

$$
E\left[v(c) \mid c \leq c_{1}+\varepsilon\right]<c_{1}
$$

so that in the infrequent trading market trade will happen only with the low types. In particular, if $\alpha$ is such that

$$
\alpha v(0)+(1-\alpha) v\left(c_{1}\right)<c_{1}
$$

then as $\varepsilon \rightarrow 0$ and $T \rightarrow \infty$, the infrequent trading equilibrium price converges to $v(0)$ and the surplus converges to

$$
\lim _{\varepsilon \rightarrow 0, T \rightarrow \infty} S_{I}=\alpha v(0)+(1-\alpha) c_{1}
$$

The equilibrium path for the continuous trading market is independent of the distribution and hence

$$
\begin{aligned}
\lim _{\varepsilon \rightarrow 0, T \rightarrow \infty} S_{C} & =\alpha v(0)+(1-\alpha)\left[e^{-r \tau\left(c_{1}\right)} v\left(c_{1}\right)+\left(1-e^{-r \tau\left(c_{1}\right)}\right) c_{1}\right] \\
& =\lim _{\varepsilon \rightarrow 0, T \rightarrow \infty} S_{I}+(1-\alpha)\left(e^{-r \tau\left(c_{1}\right)}\left(v\left(c_{1}\right)-c_{1}\right)\right)
\end{aligned}
$$

where $\tau(k)$ is the inverse of the function $k_{t}$. The last term is strictly positive for any $c_{1}<v\left(c_{1}\right)$. In particular, as we show in Appendix B, with $v(c)=\frac{1+c}{2}, e^{-r \tau(c)}=(1-c)$ and $v\left(c_{1}\right)-c_{1}=\frac{1}{2}\left(1-c_{1}\right)$, so

$$
\lim _{\varepsilon \rightarrow 0, T \rightarrow \infty} S_{C}=\lim _{\varepsilon \rightarrow 0, T \rightarrow \infty} S_{I}+\frac{1}{2}(1-\alpha)\left(1-c_{1}\right)^{2} .
$$

Proof of Proposition 4. In this case the equilibrium conditions (12), (13) and (14) simplify to

$$
\begin{aligned}
\frac{1}{2}+\frac{k_{t^{*}}+k_{T-\Delta}}{4} & =p_{T-\Delta} \\
\left(1-e^{-r \Delta}\right) k_{T-\Delta}+\left(\frac{1}{2}+\frac{k_{T-\Delta}}{2}\right) e^{-r \Delta} & =p_{T-\Delta} \\
\left(1-e^{-r \Delta_{2}}\right) k_{t^{*}}+e^{-r \Delta_{2}} p_{T-\Delta} & =\frac{1}{2}+\frac{k_{t^{*}}}{2}
\end{aligned}
$$

where $\Delta_{2}=T-\Delta-t^{*}$. 
The solution of the first two equations is:

$$
\begin{aligned}
& k_{T-\Delta}=\frac{k_{t^{*}}+2-2 e^{-r \Delta}}{3-2 e^{-r \Delta}} \\
& p_{T-\Delta}=\frac{1}{2}\left(\frac{2-e^{-r \Delta}}{3-2 e^{-r \Delta}} k_{t^{*}}+\frac{4-3 e^{-r \Delta}}{3-2 e^{-r \Delta}}\right)
\end{aligned}
$$

Substituting the price to the last condition yields

$$
\left(1-e^{-r \Delta_{2}}\right) k_{t^{*}}+e^{-r \Delta_{2}}\left(\frac{1}{2}\left(\frac{2-e^{-r \Delta}}{3-2 e^{-r \Delta}} k_{t^{*}}+\frac{4-3 e^{-r \Delta}}{3-2 e^{-r \Delta}}\right)\right)=\frac{1}{2}+\frac{k_{t^{*}}}{2}
$$

which can be solved for $\Delta_{2}$ independently of $k_{t^{*}}$ (given our assumptions about $v(c)$ and $F(c)$ ).

$$
r \Delta_{2}=-\ln \frac{3-2 e^{-r \Delta}}{4-3 e^{-r \Delta}}
$$

Note that

$$
\lim _{\Delta \rightarrow 0} \frac{\partial \Delta_{2}}{\partial \Delta}=\lim _{\Delta \rightarrow 0} \frac{\partial}{\partial \Delta} \frac{1}{r}\left(-\ln \frac{3-2 e^{-r \Delta}}{4-3 e^{-r \Delta}}\right)=1
$$

so $\Delta_{2}$ is approximately equal to $\Delta$.

In the continuous trading design, $\Omega_{C}$, cutoffs follow $k_{t}=1-e^{-r t}, \dot{k}_{t}=r e^{-r t}$. Normalize $T=1$ (and re-scale $r$ appropriately). Then

$$
k_{t^{*}}=1-e^{-r\left(1-\Delta-\Delta_{2}\right)}=1-\frac{4-3 e^{-r \Delta}}{3-2 e^{-r \Delta}} e^{r \Delta} \delta
$$

where $\delta=e^{-r}$ and

$$
t^{*}=1-\Delta-\Delta_{2}=1-\Delta+\frac{1}{r} \ln \frac{3-2 e^{-r \Delta}}{4-3 e^{-r \Delta}}
$$

We can now compare gains from trade in the two cases. The surplus starting at time $t^{*}$ is (including discounting):

$$
\begin{aligned}
S_{c}(\Delta) & =\int_{k_{t *}}^{1-e^{-r}} e^{-r \tau(c)}(v(c)-c) d c+\delta \int_{1-e^{-r}}^{1}(v(c)-c) d c \\
& =\int_{k_{t^{*}}}^{1-e^{-r}}(1-c)\left(\frac{1-c}{2}\right) d c+\delta \int_{1-e^{-r}}^{1}\left(\frac{1-c}{2}\right) d c
\end{aligned}
$$

where we used $e^{-r \tau(c)}=1-c$.

$$
\frac{\partial S_{c}(\Delta)}{\partial \Delta}=-\frac{\partial k_{t^{*}}}{\partial \Delta} \frac{\left(1-k_{t^{*}}\right)^{2}}{2}
$$

and since $\lim _{\Delta \rightarrow 0} \frac{\partial k_{t^{*}}}{\partial \Delta}=-2 r \delta$ we get that

$$
\lim _{\Delta \rightarrow 0} \frac{\partial S_{c}(\Delta)}{\partial \Delta}=r \delta^{3}
$$

For the "late closure" market the gains from trade are

$$
S_{L C}(\Delta)=e^{-r(1-\Delta)} \int_{k_{t^{*}}}^{k_{T-\Delta}}(v(c)-c) d c+e^{-r} \int_{k_{T-\Delta}}^{1}(v(c)-c) d c
$$

after substituting the computed values for $k_{t^{*}}$ and $k_{T-\Delta}$ it can be verified that

$$
\lim _{\Delta \rightarrow 0} \frac{\partial S_{L C}(\Delta)}{\partial \Delta}=r \delta^{3}
$$

which is the same as in the case of continuous market, so to the first approximation even conditional on reaching $t^{*}$ the gains from trade are approximately the same in the two market designs. 
We can compare the second derivatives:

$$
\begin{gathered}
\lim _{\Delta \rightarrow 0} \frac{\partial^{2} S_{L C}(\Delta)}{\partial^{2} \Delta}=3 \delta^{3} r^{2} \\
\lim _{\Delta \rightarrow 0} \frac{\partial^{2} S_{C}(\Delta)}{\partial^{2} \Delta}=3 \delta^{3} r^{2}
\end{gathered}
$$

and even these are the same. Finally, comparing third derivatives:

$$
\begin{aligned}
& \lim _{\Delta \rightarrow 0} \frac{\partial^{3} S_{L C}(\Delta)}{\partial^{3} \Delta}=13 r^{3} \delta^{3} \\
& \lim _{\Delta \rightarrow 0} \frac{\partial^{3} S_{C}(\Delta)}{\partial^{3} \Delta}=9 r^{3} \delta^{3}
\end{aligned}
$$

so we get that for small $\Delta$, the "late closure" market generates slightly higher expected surplus, but the effects are really small.

Proof of Theorem 2. Let's consider first the equilibrium objects at times the market is open conditional on the seller having arrived:

For prices:

$$
p_{t}=E\left[v(c) \mid c \in\left[k_{t-\Delta}, k_{t}\right]\right]
$$

For cutoffs:

$$
\left(p_{t}-k_{t}\right)=e^{-r \Delta}\left(p_{t+\Delta}-k_{t}\right)
$$

Let $v(c)=\alpha c+(1-\alpha) F(c)=c$, so that these conditions become:

$$
\begin{aligned}
& p_{t}=\left((1-\alpha)+\alpha \frac{k_{t-\Delta}+k_{t}}{2}\right) \\
& \left(\left((1-\alpha)+\alpha \frac{k_{t-\Delta}+k_{t}}{2}\right)-k_{t}\right)=e^{-r \Delta}\left(\left((1-\alpha)+\alpha \frac{k_{t+\Delta}+k_{t}}{2}\right)-k_{t}\right)
\end{aligned}
$$

Combining these two and rearranging, we get a second-order linear difference equation for $k_{t}$ :

$$
2(1-\alpha)\left(1-e^{-r \Delta}\right)=e^{-r \Delta} \alpha k_{t+\Delta}+(2-\alpha)\left(1-e^{-r \Delta}\right) k_{t}-\alpha k_{t-\Delta}
$$

Guess $k_{t}=A k_{t-1}+B$.

For $k_{t} \rightarrow 1$ we must have

$$
B+A B+A^{2} B+\ldots=1 \Rightarrow B=1-A .
$$

Plugging-in our guess to (7) we get

$$
\begin{aligned}
2(1-\alpha)\left(1-e^{-r \Delta}\right)= & e^{-r \Delta} \alpha\left(\left(A\left(A k_{t-\Delta}+(1-A)\right)+(1-A)\right)\right)+ \\
& (2-\alpha)\left(1-e^{-r \Delta}\right)\left(A k_{t-\Delta}+(1-A)\right)-\alpha k_{t-\Delta}
\end{aligned}
$$

Since this must hold for all $t$, we must have:

$$
0=e^{-r \Delta} \alpha A^{2}+(2-\alpha)\left(1-e^{-r \Delta}\right) A-\alpha
$$

Solving for $A$ we get:

$$
A=\left(\frac{-(2-\alpha)\left(1-e^{-r \Delta}\right)+\sqrt{\left[(2-\alpha)\left(1-e^{-r \Delta}\right)\right]^{2}+4 e^{-r \Delta} \alpha^{2}}}{2 e^{-r \Delta} \alpha}\right) .
$$

This implies that for any $t \in\{0, \Delta, \ldots\}$

$$
k_{t}=1-\left(\frac{-(2-\alpha)\left(1-e^{-r \Delta}\right)+\sqrt{\left[(2-\alpha)\left(1-e^{-r \Delta}\right)\right]^{2}+4 e^{-r \Delta} \alpha^{2}}}{2 e^{-r \Delta} \alpha}\right)^{t} .
$$


Next we need to calculate welfare. As a first step, calculate the surplus assuming the seller is present at time 0 :

$$
\begin{aligned}
s(\Delta) & =\sum_{j=0}^{\infty} e^{-r \Delta j}\left(\int_{k_{j}}^{k_{j+1}}(v(c)-c) d c\right) \\
& =\sum_{j=0}^{\infty} e^{-r \Delta j}\left(\int_{k_{j}}^{k_{j+1}}((1-\alpha)(1-c)) d c\right) .
\end{aligned}
$$

Let $\delta=e^{-r \Delta}$ to express the surplus as:

$$
s(\delta)=\left(\frac{1-\alpha}{2}\right) \sum_{n=0}^{\infty} \delta^{n}\left(k_{n+1}-k_{n}\right)\left(2-\left(k_{n}+k_{n+1}\right)\right) .
$$

Let

$$
G(\delta, \alpha) \equiv\left(\frac{-(2-\alpha)(1-\delta)+\sqrt{[(2-\alpha)(1-\delta)]^{2}+4 \delta \alpha^{2}}}{2 \delta \alpha}\right)
$$

and

$$
X(\delta, \alpha) \equiv(G(\delta, \alpha))^{2}
$$

We can then simplify the expression for the conditional surplus to:

$$
s(\delta)=\left(\frac{1-\alpha}{2}\right) \frac{1-X(\delta, \alpha)}{1-\delta X(\delta, \alpha)}
$$

As the second step, we need to calculate the expected present value of that surplus. Given an arrival at time $t \in(0, \Delta)$ the discount factor is $e^{-r(\Delta-t)}$. Given that the arrival is governed by a Poisson process, the expected discount factor is:

$$
\int_{0}^{\Delta} \frac{e^{-r(\Delta-t)} \lambda e^{-\lambda t}}{1-e^{-\lambda \Delta}} d t
$$

So the unconditional surplus is:

$$
S\left(\Omega_{\Delta}\right)=\left(\int_{0}^{\Delta} \frac{e^{-r(\Delta-t)} \lambda e^{-\lambda t}}{1-e^{-\lambda \Delta}} d t\right)\left(\frac{1-\alpha}{2}\right) \frac{1-X(\delta, \alpha)}{1-\delta X(\delta, \alpha)}
$$

Although the analytical expressions are a bit cumbersome, one can easily compute numerically the difference in surplus between continuous trading and trading at intervals of time $\Delta$ to show that this difference is positive and increasing in $\Delta$ for all $\alpha \in(0,1), \lambda>0$ and $\Delta>0$.

\section{Appendix B. Computing equilibria for continuous and infrequent trading}

Infrequent trading The infrequent trading market design corresponds to the classic market for lemons as in Akerlof (1970). The equilibrium in this case is described by a price $p_{0}$ and a cutoff $k_{0}$ that satisfy that the cutoff type is indifferent between trading at $t=0$ and waiting till $T$ :

$$
p_{0}=(1-\delta) k_{0}+\delta \frac{1+k_{0}}{2}
$$

and that the buyers break even on average:

$$
p_{0}=E\left[v(c) \mid c \leq k_{0}\right]
$$

The solution is $k_{0}=\frac{2-2 \delta}{3-2 \delta}$ and $p_{0}=\frac{4-3 \delta}{6-4 \delta}$. The expected gains from trade are

$$
S_{I}=\int_{0}^{k_{0}}(v(c)-c) d c+\delta \int_{k_{0}}^{1}(v(c)-c) d c=\frac{4 \delta^{2}-11 \delta+8}{4(2 \delta-3)^{2}}
$$


With infrequent trading, $\Omega_{I}$, for general $f$ and $v$ we have the following characterization of equilibria ${ }^{15}$ :

Proposition 2 (Infrequent/Restricted Trading). For $\Omega_{I}=\{0, T\}$ there exists a competitive equilibrium $\left\{p_{0}, k_{0}\right\}$. Equilibria are a solution to:

$$
\begin{aligned}
& p_{0}=E\left[v(c) \mid c \in\left[0, k_{0}\right]\right] \\
& p_{0}=\left(1-e^{-r T}\right) k_{0}+e^{-r T} v\left(k_{0}\right)
\end{aligned}
$$

If $\frac{f(c)}{F(c)}(v(c)-c)-\frac{\delta}{1-\delta} v^{\prime}(c)$ is strictly decreasing, the equilibrium is unique.

Proof of Proposition 2. 1) Existence. The equilibrium conditions follow from the definition of equilibrium. To see that there exists at least one solution to (8) and (9) note that if we write the condition for the cutoff as:

$$
E\left[v(c) \mid c \leq k_{0}\right]-\left(\left(1-e^{-r T}\right) k_{0}+e^{-r T} v\left(k_{0}\right)\right)=0
$$

then the LHS is continuous in $k_{0}$, it is positive at $k_{0}=0$ and negative at $k_{0}=1$. So there exists at least one solution. ${ }^{16}$

2) Uniqueness. To see that there is a unique solution under the two assumptions, note that the derivative of the LHS of (10) at any $k$ is

$$
\frac{f(k)}{F(k)}(v(k)-E[v(c) \mid c \leq k])-(1-\delta)-\delta v^{\prime}(k)
$$

When we evaluate it at points where (10) holds, the derivative is

$$
\frac{f(k)}{F(k)}(v(k)-k)(1-\delta)-(1-\delta)-\delta v^{\prime}(k)
$$

and that is by assumption decreasing in $k$.

Suppose that there are at least two solutions and select two: the lowest $k_{L}$ and second-lowest $k_{H}$. Since $k_{L}$ is the lowest solution, at that point the curve on the LHS of (10) must have a weakly negative slope (since the curve crosses zero from above). However, our assumption implies that curve has even strictly more negative slope at $k_{H}$. That leads to a contradiction since by assumption between $\left[k_{L}, k_{H}\right]$ the LHS is negative, so with this ranking of derivatives it cannot become 0 at $k_{H}$

Continuous trading The above outcome cannot be sustained in equilibrium if there are multiple occasions to trade before $T$. If at $t=0$ types below $k_{0}$ trade, the next time the market opens price would be at least $v\left(k_{0}\right)$. If so, types close to $k_{0}$ would be strictly better off delaying trade. As a result, for any set $\Omega$ richer than $\Omega_{I}$, in equilibrium there is less trade in period 0 .

If we look at the case of continuous trading, $\Omega_{C}=[0, T]$, then the equilibrium with continuous trade is a pair of two processes $\left\{p_{t}, k_{t}\right\}$ that satisfy:

$$
\begin{aligned}
p_{t} & =v\left(k_{t}\right) \\
r\left(p_{t}-k_{t}\right) & =\dot{p}_{t}
\end{aligned}
$$

The intuition is as follows. Since the process $k_{t}$ is continuous, the zero profit condition is that the price is equal to the value of the current cutoff type. The second condition is the indifference of the current cutoff type between trading now and waiting for a $d t$ and trading at a higher price. These conditions yield a differential equation for the cutoff type

$$
r\left(v\left(k_{t}\right)-k_{t}\right)=v^{\prime}\left(k_{t}\right) \dot{k}_{t}
$$

with the boundary condition $k_{0}=0$. In our example it has a simple solution:

$$
k_{t}=1-e^{-r t}
$$

The total surplus from continuous trading is

$$
S_{C}=\int_{0}^{T} e^{-r t}\left(v\left(k_{t}\right)-k_{t}\right) \dot{k}_{t} d t+e^{-r T} \int_{k_{T}}^{1}(v(c)-c) d c=\frac{1}{12}\left(2+\delta^{3}\right) .
$$

\footnotetext{
15 The infrequent trading model is the same as the model in Akerlof (1970) if $T=\infty$.

16 If there are multiple solutions, a game theoretic-model would refine some of them, see section 13.B of Mas-Colell, Whinston and Green (1995) for a discussion.
} 
For general $f$ and $v$, with continuous trading opportunities $\Omega_{C}$, the equilibrium is unique:

Proposition 3 (Continuous trading). For $\Omega_{C}=[0, T]$ a competitive equilibrium (unique up to measure zero of times) is the unique solution to:

$$
\begin{aligned}
p_{t} & =v\left(k_{t}\right) \\
k_{0} & =0 \\
r\left(v\left(k_{t}\right)-k_{t}\right) & =v^{\prime}\left(k_{t}\right) \dot{k}_{t}
\end{aligned}
$$

Proof of Proposition 3. This proof follows very closely the proof of Proposition 1 in FS.

First note that our requirement $p_{t} \geq v\left(k_{t-}\right)$ implies that there cannot be any atoms of trade, i.e., that $k_{t}$ has to be continuous. Suppose not, that at time $s$ types $\left[k_{s-}, k_{s}\right]$ trade with $k_{s_{-}}<k_{s}$. Then at time $s+\varepsilon$ the price would be at least $v\left(k_{s}\right)$ while at $s$ the price would be strictly smaller to satisfy the zero-profit condition. But then for small $\varepsilon$ types close to $k_{s}$ would be better off not trading at $s$, a contradiction. Therefore we are left with processes such that $k_{t}$ is continuous and $p_{t}=v\left(k_{t}\right)$. For $k_{t}$ to be strictly increasing over time we need that $r\left(p_{t}-k_{t}\right)=\dot{p}_{t}$ for almost all $t$ : if the price was rising faster, current cutoffs would like to wait, a contradiction. If prices were rising slower over any time interval starting at $s$, there would be an atom of types trading at $s$, another contradiction. So the only remaining possibility is that $\left\{p_{t}, k_{t}\right\}$ are constant over some interval $\left[s_{1}, s_{2}\right]$. Since the price at $s_{1}$ is $v\left(k_{s_{1-}}\right)$ and the price at $s_{2}$ is $v\left(k_{s_{2}}\right)$, we would obtain a contradiction that there is no atom of trade in equilibrium. In particular, if $p_{s_{1}}=p_{s_{2}}$ (which holds if and only if $k_{s_{1-}}=k_{s_{1}}=k_{s_{2}}$ ) then there exist types $k>k_{s_{1}}$ such that

$$
v\left(k_{s_{1}}\right)>\left(1-e^{r\left(s_{2}-s_{1}\right)}\right) k+e^{r\left(s_{2}-s_{1}\right)} v\left(k_{s_{1}}\right)
$$

and these types would strictly prefer to trade at $t=s_{1}$ than to wait till $s_{2}$, a contradiction again.

Remark 2. In this paper we analyze competitive equilibria. In this benchmark example it is possible to write a gametheoretic version of the model allowing two buyers to make public offers every time the market is open. If we write the model having $\Omega=\{0, \Delta, 2 \Delta, \ldots, T\}$ then we can show that there is a unique Perfect Bayesian Equilibrium for every $T$ and $\Delta>0$. When $\Delta=T$ then the equilibrium coincides with the equilibrium in the infrequent trading market we identify above. Moreover, taking the sequence of equilibria as $\Delta \rightarrow 0$, the equilibrium path converges to the competitive equilibrium we identify for the continuous trading design. In other words, the equilibria we describe in this section have a game-theoretic foundation.

\section{Appendix C. Extensions and discussion}

\section{C.1. Closing the market briefly before information arrives}

The final design we consider is the possibility of keeping the market open continuously from $t=0$ till $T-\Delta$ and then closing it till $T$. Such a design seems realistic and in some practical situations may be easier to implement than $\Omega^{E C}$. It may be easier to determine when some private information is expected to arrive (i.e., when $t=T$ ) than when it is that the seller of the asset is hit by liquidity needs (i.e., when $t=0$ ).

The comparison of this "late closure" market with the continuous trading market is much more complicated than in Section 3.2 for two related reasons. First, if the market is closed from $T-\Delta$ to $T$, there will be an atom of types trading at $T-\Delta$. As a result, there will be a "quiet period" before $T-\Delta$ : there will be some time interval $\left[t^{*}, T-\Delta\right]$ such that despite the market being open, no types will trade on the equilibrium path in that interval. The equilibrium outcome until $t^{*}$ is the same in the "late closure" as in the continuous trading design, but diverges from that point on. That brings the second complication: starting at time $t^{*}$, the continuous trading market benefits from some types trading earlier than in the "late closure" market. Therefore it is not sufficient to show that by $T$ there are more types that trade in the late closure market. We actually have to compare directly the total surplus generated between $t^{*}$ and $T$. These two complications are not present when we consider the "early closure" design since there is no $t^{*}$ before $t=0$.

An equilibrium in the "late closure" design is as follows. Let $p_{T-\Delta}^{*}, k_{T-\Delta}^{*}$ and $t^{*}$ be a solution to the following system of equations:

$$
\begin{aligned}
E\left[v(c) \mid c \in\left[k_{t^{*}}, k_{T-\Delta}\right]\right] & =p_{T-\Delta} \\
\left(1-e^{-r \Delta}\right) k_{T-\Delta}+e^{-r \Delta} v\left(k_{T-\Delta}\right) & =p_{T-\Delta} \\
\left(1-e^{-r\left(T-\Delta-t^{*}\right)}\right) k_{t^{*}}+e^{-r\left(T-\Delta-t^{*}\right)} p_{T-\Delta} & =v\left(k_{t^{*}}\right)
\end{aligned}
$$




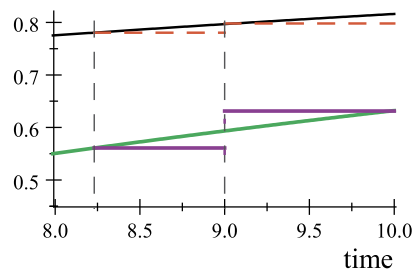

Fig. 6. Late Closure path of prices and cutoffs in case $T=10, \Delta=1, r=0.1, v(c)=\frac{c+1}{2}$, and $F(c)=c$.

where the first equation is the zero-profit condition at $t=T-\Delta$, the second equation is the indifference condition for the highest type trading at $T-\Delta$ and the last equation is the indifference condition of the lowest type that reaches $T-\Delta$, who chooses between trading at $t^{*}$ and at $T-\Delta$. The equilibrium for the late closure market is then:

1) at times $t \in\left[0, t^{*}\right],\left(p_{t}, k_{t}\right)$ are the same as in the continuous trading market

$2)$ at times $t \in\left(t^{*}, T-\Delta\right),\left(p_{t}, k_{t}\right)=\left(v\left(k_{t^{*}}\right), k_{t^{*}}\right)$

3) at $t=T-\Delta,\left(p_{t}, k_{t}\right)=\left(p_{T-\Delta}^{*}, k_{T-\Delta}^{*}\right)$

Condition (14) guarantees that given the constant price at times $t \in\left(t^{*}, T-\Delta\right)$ it is indeed optimal for the seller not to trade. There are other equilibria that differ from this equilibrium in terms of the prices in the "quiet period" time: any price process that satisfies in this time period

$$
\left(1-e^{-r(T-\Delta-t)}\right) k_{t^{*}}+e^{-r(T-\Delta-t)} p_{T-\Delta} \geq p_{t} \geq v\left(k_{t^{*}}\right)
$$

satisfies all our equilibrium conditions. Yet, all these paths yield the same equilibrium outcome in terms of trade and surplus (of course, the system (12)-(14) may have multiple solutions that would have different equilibrium outcomes).

Despite this countervailing inefficiency, for our leading linear-uniform example:

Proposition 4. Suppose $v(c)=\frac{1+c}{2}$ and $F(c)=c$. For every $r$ and $T$ there exists a $\Delta>0$ such that the "late closure" market design, $\Omega^{L C}=[0, T-\Delta] \cup\{T\}$, generates higher expected gains from trade than the continuous trading market, $\Omega_{C}$. Yet, for small $\Delta$, the gains from late closure are smaller than the gains from early closure.

The proof (in the Appendix) shows third-order gains of welfare from the late closure, while the gains from early closure are first-order. Fig. 6 illustrates the reason the gains from closing the market are small relative to when the market is closed at time zero. The bottom two lines show the evolution of the cutoff type in $\Omega_{C}$ (continuous curve) and in $\Omega^{L C}$ (discontinuous at $t=T-\Delta=0.9$ ). The top two lines show the corresponding path of prices. The gains from bringing forward trades that occur when the market is exogenously closed in $t \in(9,10)$ (i.e., the jump in types at $t=0.9)$ are partially offset by the delay of types in the endogenous quiet period $t \in(8.23,9)$. If we close the market for $t \in(0, \Delta)$ instead, there is no loss from some types postponing trade because there is no time before 0 .

The intuition why the gains (if any) are in general very small is that we prove that the endogenous quiet period is approximately of the length $\Delta$ (up to first-order approximation at $\Delta$ close to zero). The reasoning in Result 1 implies that the jump in types at time $T-\Delta$ is approximately twice as large as the continuous increase in the cutoff when the market is opened continuously over a time interval of length $\Delta$. Putting these two observations together implies that the final cutoff at time $T$ is approximately (using a first-order approximation in $\Delta$ ) the same for these two designs, as seen in Fig. 6. Hence, any welfare effects are tiny.

\section{C.2. Stochastic arrival of information}

So far we have assumed that it is known that the private information is revealed at $T$. However, in some markets, the market participants may be uncertain about the timing of its revelation even if the private information is short-lived.

To capture that idea we have analyzed a version of our model in which there is no fixed time $T$ when information is revealed, so that $\Omega \subseteq[0, \infty]$, but over time exogenous information that publicly reveals the seller's type arrives with a constant Poisson rate $\lambda$. We assume that for any $\Omega$ trade is immediate at price $p=v(c)$ once the information arrives.

The following results can be established for this model:

1. If $\Omega=[0, \infty]$, then for all $\lambda$ the equilibrium is as described in Proposition 3 .

2. If $\frac{f(c)}{F(c)} \frac{v(c)-c}{\lambda v^{\prime}(c)+r}$ is decreasing, then the analog of Theorem 1 holds (i.e., with this new condition replacing the regularity condition, fully restricting trade between $t=0$ and the time information is revealed maximizes total surplus).

The proofs of these claims are analogous to the proofs in the original and hence are omitted (but they are available from the authors upon request). The intuition for why the equilibrium path of prices and cutoffs before information arrives is the same in the stochastic and deterministic arrival of information cases is as follows. In the deterministic case, the benefit of 
delaying trade by $d t$ is that the price increases by $\dot{p}_{t} d t$. In the stochastic case, the price also increases, but additionally with probability $\lambda d t$ the news arrives. If so, the current cutoff type gets a price $v\left(k_{t}\right)$ instead of $p_{t+d t}$. However, since $p_{t}=v\left(k_{t}\right)$, price $p_{t+d t}$ is only of order $d t$ higher than what the current cutoff gets upon arrival. Therefore, the additional effect of delaying trade is a term on the order of $d t^{2}$. So with continuous trading stochastic arrival of information does not affect incentives to delay, and the equilibrium path of cutoffs is unchanged.

To illustrate the model with random arrival, return to our benchmark example with $v(c)=\frac{1+c}{2}$ and $F(c)=c$. In the infrequent trading market, the equilibrium $\left(p_{0}, k_{0}\right)$ is determined by:

$$
\begin{aligned}
& p_{0}=\frac{\lambda}{\lambda+r} v\left(k_{0}\right)+\frac{r}{\lambda+r} k_{0} \\
& p_{0}=E\left[v(c) \mid c \leq k_{0}\right]
\end{aligned}
$$

where the first equation is the indifference condition of the cutoff type and the second equation is the usual zero-profit condition. In our example we get

$$
k_{0}=\frac{2 r}{3 r+\lambda}, p_{0}=\frac{4 r+\lambda}{6 r+2 \lambda}
$$

We now can compare the gains from trade. The total gains from trade in the infrequent trading market are:

$$
S_{I}=\int_{0}^{k_{0}}(v(c)-c) d c+\frac{\lambda}{\lambda+r} \int_{k_{0}}^{1}(v(c)-c) d c .
$$

In the continuous trading market (since the path of types is the same as we computed before) the gains are:

$$
S_{C}=\int_{0}^{+\infty} \lambda e^{-\lambda t}\left(\int_{0}^{k_{t}} e^{-r \tau(c)}(v(c)-c) d c+e^{-r t} \int_{k_{t}}^{1}(v(c)-c) d c\right) d t
$$

where $\tau(c)=-\frac{\ln (1-c)}{r}$ is the time type $c$ trades if there is no arrival before $\tau(c)$. Direct calculations yield:

$$
S_{I}(z)-S_{C}(z)=\frac{1}{2}(z+3)^{-2}>0
$$

where $z \equiv \frac{\lambda}{r}$. So, for every $\lambda$, the infrequent trading market is more efficient than the continuous trading market, consistent with the analog of Theorem 1.

\section{C.3. Beyond design of $\Omega$ : affecting $T$}

In this paper we analyze different choices of $\Omega$. A natural question is what else could a market designer affect to improve market efficiency. One such possibility is information structure, as we have discussed in Remark 1. There are of course other options for changing information (for example, should past rejected offers be observed or not?), but that is beyond the scope of this paper.

Another possibility is changing $T$. Clearly, if the market designer could make $T$ very small, it would be good for welfare since it would make the market imperfections short-lived. That may not be feasible though. Suppose instead that the designer could only increase $T$ (for example, by making some verification take longer). ${ }^{17}$ Surprisingly, it turns out that in some cases increasing $T$ could improve efficiency. While it is never beneficial in the continuous-trading case (since it does not affect trade before $T$ and only delays subsequent trades), it can help in other cases. To illustrate it, Fig. 7 graphs the expected gains from trade in our leading example for $\Omega_{I}=\{0, T\}$ as a function of $\delta=e^{-r T}$. It turns out that if and only if $e^{-r T}<\frac{1}{2}$, increasing $T$ is welfare improving.

\section{C.4. Common knowledge of gains from trade}

We have assumed that $v(0)>0$ and $v(1)=1$, that is, strictly positive gains from trade for the lowest type and no gains on the top. Can we relax these assumptions?

17 We thank Marina Halac for suggesting this question. 


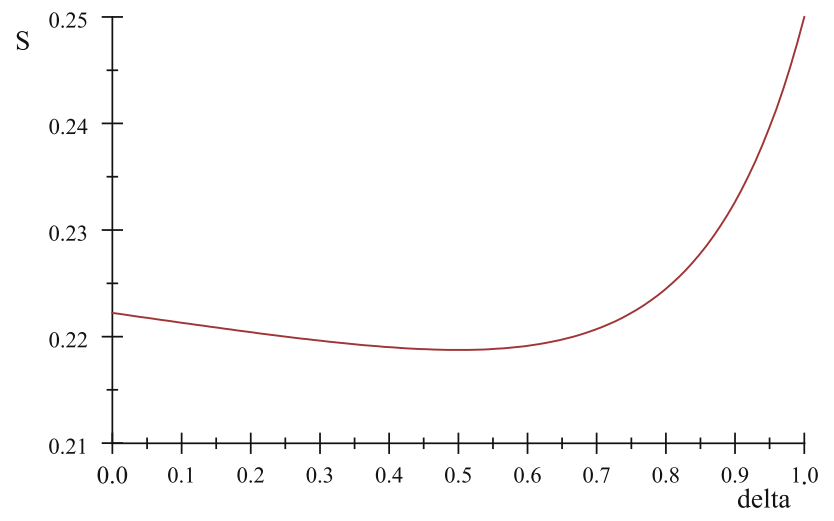

Fig. 7. Surplus with infrequent trading as a function of $T$.

\section{C.4.1. Role of $v(0)>0$}

If $v(0)=0$ then Theorem 1 still applies. As we argued above, if the market is opened continuously, in equilibrium there is no trade before $T$ (to see this note that the starting price would leave the lowest type with no surplus, so that type would always prefer to wait for a price increase). That does not need to be true for other $\Omega$. For example, if $v(c)=\sqrt{c}$ and $F(c)=c$, then for all $T$ the conditions in Theorem 1 are satisfied. Therefore, $\Omega_{I}=\{0, T\}$ is welfare-maximizing and $\Omega_{C}=[0, T]$ is welfare-minimizing over all $\Omega$; and if $\delta<\frac{2}{3}$ then the ranking is strict since there is some trade with $\Omega_{I}$.

\section{C.4.2. Role of $v(1)=1$}

The main reason we assume $v(1)=1$ is that in this way we do not need to define equilibrium market prices after histories where the seller trades with probability 1 . That is, when $v(1)=1$, the highest type never trades in equilibrium no matter how large is $T$. This makes our definition of competitive equilibrium simpler than in Daley and Green (2012) (compare our condition (3) "Market Clearing" with Definition 2.1 there).

To illustrate how the freedom in selecting off-equilibrium-path beliefs can lead to a multiplicity of equilibria with radically different outcomes, consider the following heuristic reasoning. Assume:

$$
F(c)=c ; v(c)=c+s
$$

Suppose that $\Omega=\{0, \Delta, 2 \Delta, \ldots, T\}$ for $\Delta>0$. Let $s>\frac{1}{2}$ so that in a static problem trade would be efficient.

Case 1: Assume that when an offer that all types accept on the equilibrium path is rejected, buyers believe the seller has the highest type, $c=1$. That is, post-rejection price is $1+s$. Then, taking a sequence of equilibria as $\Delta \rightarrow 0$, we can show that in the limit trade is smooth over time (no atoms) with:

$$
\begin{aligned}
p_{t}(k) & =v\left(k_{t}\right) \\
k_{t} & =r s t
\end{aligned}
$$

On equilibrium path all types trade by:

$$
\tau=\frac{1}{r s}
$$

unless $\tau<T$. If the last offer, $p_{\tau}=1+s$ is rejected, the price stays constant after that, consistently with the beliefs and competition.

Case 2: Alternatively, assume that when an offer that all types accept on the equilibrium path is rejected, buyers do not update their beliefs. That is, after that history they believe the seller type is distributed uniformly over $\left[k_{t}, 1\right]$, where $k_{t}$ is derived from the history of the game. In that case we can construct the following equilibrium for all $\Delta>0$. At $t=0$ there is an initial offer $p_{0}=\frac{1}{2}+s$ and all types trade. If that initial offer is rejected, the buyers believe $c \sim U[0,1]$ and continue to offer $p_{t}=p_{0}$ for all $t>0$ (and again all types trade). This is indeed an equilibrium since the buyers break even at time zero (and at all future times given their beliefs) and no seller type is better off by rejecting the initial offer.

These equilibria are radically different in terms of efficiency: only the second one is efficient. It is beyond the scope of this paper to study in what situations or under what model extensions this multiplicity could be resolved and how. $v(1)>1$ creates similar problems for large $T$ even if immediate efficient trade is not possible. On the other hand, if the gap on top is small so that for a given $T$ in equilibrium it is not possible that all types trade before $T$, our analysis still applies.

\section{References}


Biais, B., Glosten, L., Spatt, C., 2005. Market microstructure: a survey of microfoundations, empirical results, and policy implications. Journal of Financial Markets 8 (2), 217-264.

Camargo, B., Lester, B., 2014. Trading dynamics in decentralized markets with adverse selection. Journal of Economic Theory 153, 534-568.

Chang, B., 2018. Adverse selection and liquidity distortion. The Review of Economic Studies 85 (1), $275-306$.

Daley, B., Green, B., 2012. Waiting for news in the market for lemons. Econometrica 80 (4), 1433-1504.

Du, S., Zhu, H., 2017. What is the optimal trading frequency in financial markets? The Review of Economic Studies 84 (2), $1606-1651$.

Fuchs, W., Skrzypacz, A., 2015. Government interventions in a dynamic market with adverse selection. Journal of Economic Theory $158,371-406$.

Fuchs, W., Öry, A., Skrzypacz, A., 2016. Transparency and distressed sales under asymmetric information. Theoretical Economics 11 (3), $1103-1144$.

Guerrieri, V., Shimer, R., 2014. Dynamic adverse selection: a theory of illiquidity, fire sales, and flight to quality. The American Economic Review 104 (7), 1875-1908

Guerrieri, V., Shimer, R., Wright, R., 2010. Adverse selection in competitive search equilibrium. Econometrica 78 (6), $1823-1862$.

Hendel, I., Lizzeri, A., Siniscalchi, M., 2005. Efficient sorting in a dynamic adverse-selection model. The Review of Economic Studies 72 (2), 467-497.

Janssen, M.C.W., Karamychev, V.A., 2002. Cycles and multiple equilibria in the market for durable lemons. Economic Theory 20 (3), $579-601$.

Janssen, M.C.W., Roy, S., 2002. Dynamic trading in a durable good market with asymmetric information. International Economic Review 43 (1), 257-282.

Kim, K., 2017. Information about sellers' past behavior in the market for lemons. Journal of Economic Theory 169, 365-399.

Kremer, I., Skrzypacz, A., 2007. Dynamic signaling and market breakdown. Journal of Economic Theory 133 (1), $58-82$.

Merrill, C.B., Nadauld, T.D., Stulz, R.M., Sherlund, S., 2012. Did Capital Requirements and Fair Value Accounting Spark Fire Sales in Distressed Mortgage-

Backed Securities? NBER Working Paper No. 18270.

Nöldeke, G., van Damme, E., 1990. Signalling in a dynamic labour market. The Review of Economic Studies 57 (1), 1-23.

Philippon, T., Skreta, V., 2012. Optimal interventions in markets with adverse selection. The American Economic Review 102 (1), 1-28.

Roy, S., 2014. Dynamic sorting in durable goods markets with buyer heterogeneity. Canadian Journal of Economics 47 (3), $1010-1031$.

Samuelson, W., 1984. Bargaining under asymmetric information. Econometrica 52 (4), 995-1005.

Spence, A.M., 1973. Job market signaling. The Quarterly Journal of Economics 87 (3), 355-374.

Swinkels, J.M., 1999. Educational signalling with preemptive offers. The Review of Economic Studies 66 (4), $949-970$.

Tirole, J., 2012. Overcoming adverse selection: how public intervention can restore market functioning. The American Economic Review 102 (1), 19-59.

Vayanos, D., 1999. Strategic trading and welfare in a dynamic market. The Review of Economic Studies 66 (2), $219-254$. 